\title{
Ready Access to the Echinopines Skeleton via Gold(I)-Catalyzed Alkoxycyclizations of Enynes
}

\author{
Ruth Dorel and Antonio M. Echavarren* \\ Institute of Chemical Research of Catalonia (ICIQ), Barcelona Institute of Science and Technology, Av. Països Catalans 16, 43007 \\ Tarragona, Spain
}

\section{Supporting Information}

ABSTRACT: The $[3,5,5,7]$ tetracyclic skeleton of echinopines has been stereoselectively accessed through a gold(I)-catalyzed alkoxycyclization of cyclopropyltethered 1,6-enynes. The key bicyclo[4.2.1] nonane core of the enyne precursors was readily assembled by means of a Co-catalyzed $[6+2]$ cycloaddition. Furthermore, the attempted alkoxycyclization of 1,5-enyne substrates revealed an uncovered cyclopropyl rearrangement that gives rise to $[3,6,5,7]$ tetracyclic structures.

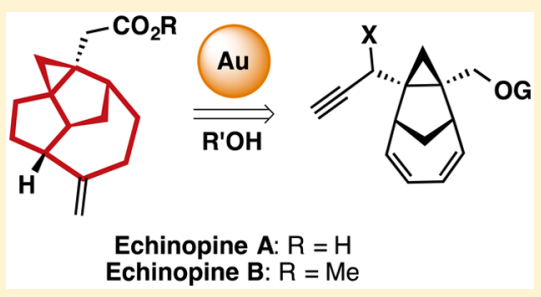

\section{INTRODUCTION}

Echinopines A and B (1 and 2) were isolated in 2008 from the roots of Echinops spinosus and feature an unprecedented $[3,5,5,7]$-membered-ring tetracyclic skeleton (Scheme 1),

Scheme 1. Strategy for the Gold-Catalyzed Synthesis of the Skeleton of Echinopines

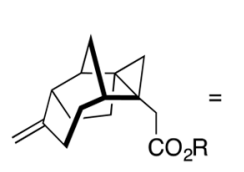

Echinopine $A(1): R=H$ Echinopine B (2): $R=M e$

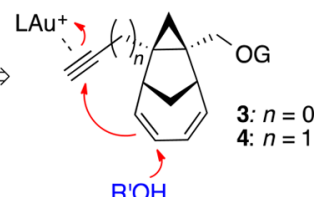

$\mathrm{R} ' \mathrm{OH}$ which probably originates biosynthetically from a guaiane precursor. $^{1}$ This complex carbon framework holds five contiguous stereogenic centers, two of them being adjacent quaternary stereocenters. Despite the fact that no biological activity has been reported to date for $\mathbf{1}$ and $\mathbf{2}$, the unique architecture of these sesquiterpenes has constituted an appealing challenge for the synthetic community and several syntheses of echinopines have been accomplished to date. ${ }^{2-7}$ The key feature in all these syntheses is the establishment of the unique $[3,5,5,7]$ skeleton, and to this aim conceptually very different ring-forming sequences have been successfully established. ${ }^{8}$ However, the assembly of the complex polycyclic framework of the echinopines skeleton is not easily addressed by conventional methods, as evidenced by the lengthy existing syntheses, and it is in most of the cases delayed to one of the last steps of the sequence.

Gold(I) catalysis constitutes a powerful tool for the construction of complex polycyclic architectures from relatively simple enyne substrates under mild reaction conditions. ${ }^{9-12} \mathrm{~A}$ concise synthesis of the complex polycyclic framework of the echinopines skeleton could easily provide access to structural analogues for further evaluation of their biological properties. In this context, we envisioned a gold(I)-catalyzed alkoxycyclization of cyclopropyl-tethered tricyclic 1,5- (3) or 1,6-enynes (4) as the key step for the ready access to the tetracyclic skeleton of echinopines via 5-endo or 5-exo cyclization, respectively (Scheme 1). ${ }^{13-16}$ This transformation would stereoselectively lead to echinopine-based tetracyclic products bearing different groups suited for further functionalization.

\section{RESULTS AND DISCUSSION}

Our approach for the synthesis of tricyclic enynes 3 and 4 relied on a cobalt-catalyzed $[6+2]$ cycloaddition between cycloheptatriene and an internal alkyne as the key step to build the bicyclo[4.2.1] nonane core. ${ }^{17,18}$ Thus, orthogonally protected diol 5 a afforded cycloadduct $6 \mathbf{a}$, which upon monodeprotection and cyclopropanation of the tetrasubstituted olefin from the less sterically hindered face gave rise to tricyclic compound 8a (Scheme 2). Oxidation of the primary alcohol and subsequent homologation employing the Ohira-Bestmann reagent provided 1,5-enyne $3 a$.

Scheme 2. Synthesis of Tricyclic 1,5-Enyne 3a

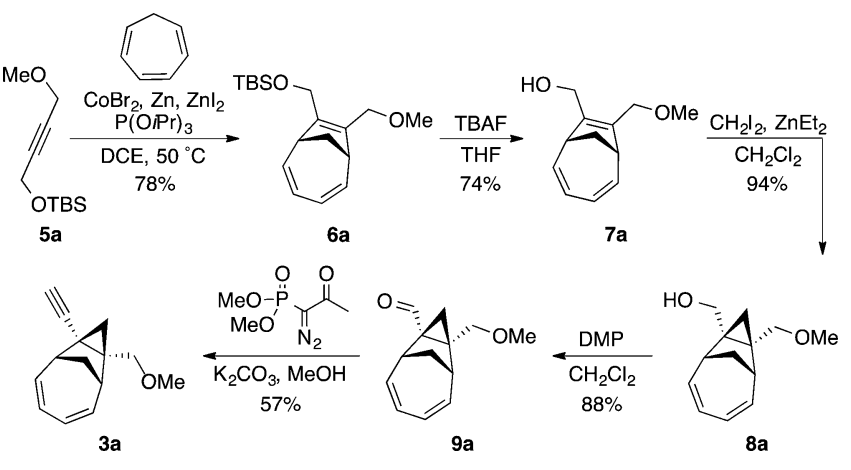

Received: July 5, 2016

Published: August 16, 2016 
Initial attempts to perform the alkoxycyclization of $3 a$ with methanol as the external nucleophile in the presence of different cationic gold(I) complexes $\mathbf{A}-\mathbf{D}$ only provided methyl ketone $\mathbf{1 0}$ as a result of the formal hydration of the terminal alkyne (Scheme 3). ${ }^{19-22}$ Moreover, when the reaction

\section{Scheme 3. Formal Hydration of 3a}
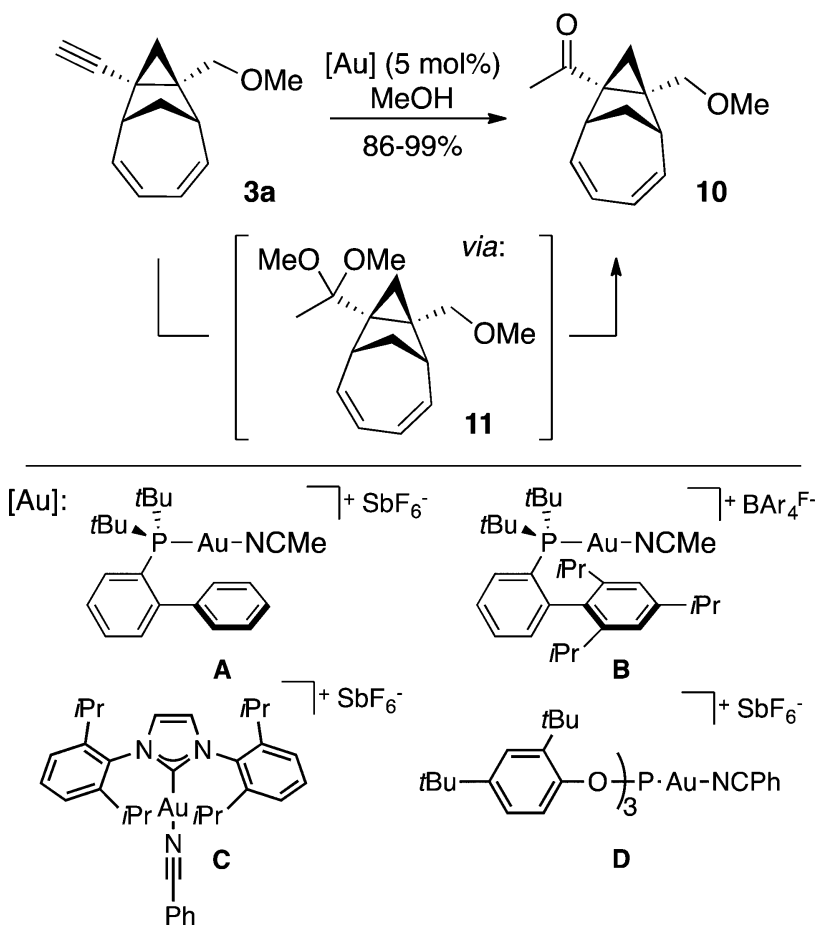

was performed under strictly anhydrous conditions, the corresponding dimethyl acetal $\mathbf{1 1}$ could be isolated, which rapidly decomposed to $\mathbf{1 0}$ under ambient conditions, thus demonstrating that the addition of methanol to the terminal alkyne of 3a is favored over the attack of the alkene moiety. Similar results were obtained when other alcohols were employed as the external nucleophiles.

The use of carbonucleophiles such as indole, 1,3-diketones, and electron-rich benzenes only resulted in the recovery of unreacted 3a. Nevertheless, when the reaction of $\mathbf{3 a}$ was performed with commercially available gold(I) complex $\mathbf{A}$ in the presence of acetic acid, complete conversion of $3 \mathbf{a}$ was achieved in $1 \mathrm{~h}$, leading to the formation of rearranged product 12 in up to $61 \%$ yield (Scheme 4). A closer mechanistic inspection of this transformation suggested that the gold(I)catalyzed reaction initially forms intermediate $\mathbf{1 4}$ that rearranges to form allyl cation $\mathbf{1 5}$, which is trapped by acetic acid. DFT calculations indicated that the formation of intermediate $\mathbf{1 6}$ that leads to $\mathbf{1 2}$ is thermodynamically favored over the formation of 17 , which is predicted to be the driving force for the rearrangement to take place. This result further illustrates the influence of the cyclopropane functionality on the reaction pathways followed in the gold(I)-catalyzed cyclizations of cyclopropane-tethered 1,5-enynes ${ }^{23}$ and underscores the propensity of the strained tetracyclic system of echinopines to undergo rearrangements. ${ }^{6}$

In order to unequivocally ensure the structure of 12 , the acetate moiety was cleaved to form alcohol 18, which was converted into the corresponding crystalline $p$-nitrobenzoate derivative 19, whose structure was confirmed by X-ray
Scheme 4. Gold-Catalyzed Rearrangement of $3 a^{a}$

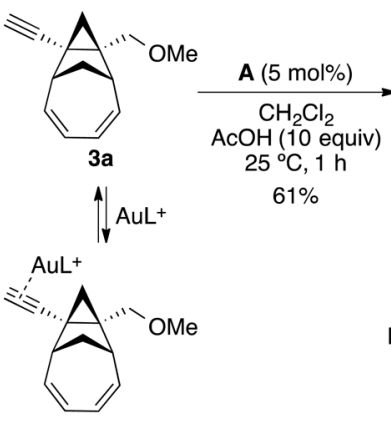

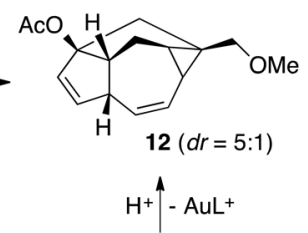

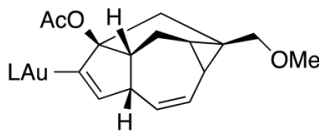
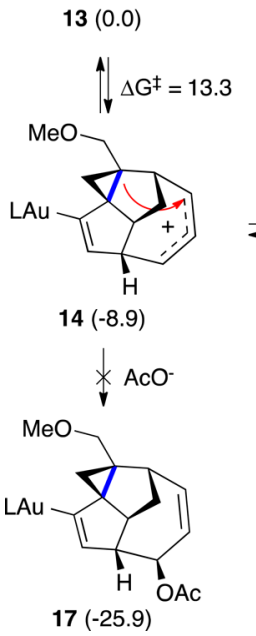

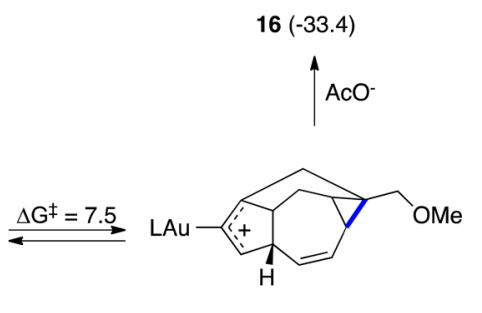

$15(-6.7)$
${ }^{a}$ Values in parentheses correspond to relative free energies in kcal $\mathrm{mol}^{-1}(\mathrm{M} 06 / 6-31 \mathrm{G}(\mathrm{d})(\mathrm{C}, \mathrm{H}, \mathrm{P}, \mathrm{O})$ and $\mathrm{SDD}(\mathrm{Au})$, solvent = $\left.\mathrm{CH}_{2} \mathrm{Cl}_{2}\right) \cdot \mathrm{L}=\mathrm{PMe}_{3}$.

diffraction (Scheme 5). ${ }^{24}$ In addition, a related system having one of the double bonds reduced was also examined with the aim of promoting a rearrangement toward the echinopine skeleton on the basis of the higher stability of carbocation $\mathbf{2 4}$ over 22 predicted by DFT calculations. Thus, 18 could be selectively hydrogenated in the presence of Crabtree's catalyst to give 20, which was converted into tertiary carbocation 22 via triflate 21. Nonetheless, the rearranged product was not observed and only nonrearranged elimination product 23 was isolated under different reaction conditions.

The synthesis of the homologous 1,6-enyne 4a commenced with the cobalt-catalyzed $[6+2]$ cycloaddition between cycloheptatriene and alkyne $\mathbf{2 5}$ followed by treatment with $\mathrm{N}$-iodosuccinimide, which afforded vinyl iodide $\mathbf{2 7}$ (Scheme 6). Kumada cross-coupling of 27 with (3-(trimethylsilyl)prop-2-yn1-yl)magnesium bromide furnished 28 , which was treated with HF.py to give allylic alcohol 29. Cyclopropanation of the tetrasubstituted olefin followed by deprotection of the terminal alkyne and protection of the primary alcohol gave rise to tricyclic 1,6-enyne 4a. However, all attempts to perform the alkoxycyclization of $\mathbf{4 a}$ in the presence of different gold(I) complexes provided only traces of the cyclized tetracyclic product and resulted in the formation of methyl ketone $\mathbf{3 1}$ as the major product.

Aldehydes $9 \mathbf{a}, \mathbf{b}^{25}$ were next employed as the platform to access a series of tricyclic 1,6-enynes featuring different functionalities at the propargylic position. Thus, the addition of ethynylmagnesium bromide provided $\mathbf{4 b , c}$ as single diastereoisomers and their alkoxycyclization was investigated 
Scheme 5. Synthesis of 23 from Acetate 12 and CYLview Depiction of the X-ray Crystal Structure of $19^{a}$

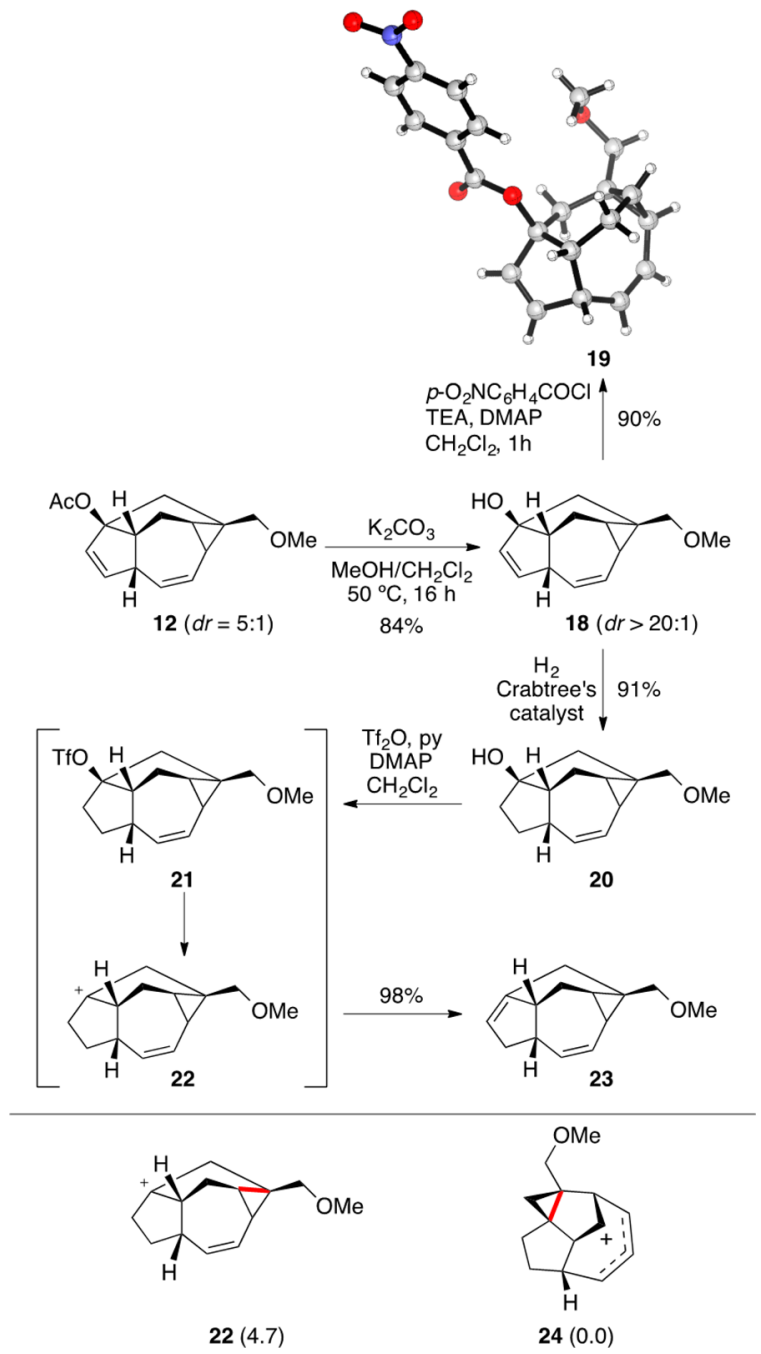

${ }^{a}$ Values in parentheses correspond to relative free energies in kcal $\mathrm{mol}^{-1}\left(\mathrm{M} 06 / 6-31 \mathrm{G}(\mathrm{d})\right.$, solvent $\left.=\mathrm{CH}_{2} \mathrm{Cl}_{2}\right)$.

using methanol as the external nucleophile in the presence of a series of gold(I) complexes spanning a range of electrophilicities. The desired alkoxycyclization products could only be detected from the reactions carried out in the presence of phosphine-gold(I) complexes, whereas gold(I) complexes bearing NHC and phosphite ligands gave complex mixtures. ${ }^{26}$ Cationic gold(I) complex B provided the best results, and the use of the alcohol as the solvent proved to be optimal for the alkoxycyclization of enynes $4 \mathbf{b}, \mathbf{c}$ to afford regio- and stereoselectively tetracyclic products $32 \mathbf{a}-\mathbf{c}$, which feature the $[3,5,5,7]$ tetracyclic skeleton of echinopines (Scheme 7). While the reaction of $\mathbf{4 b}$ with methanol provided $32 \mathrm{a}$ as a single regioisomer, the analogous reaction of $4 \mathbf{c}$ gave rise to a 5:1 mixture of regioisomers. Nonetheless, changing the external nucleophile from methanol to allyl alcohol in the reaction of $4 \mathrm{c}$ resulted in the exclusive formation of $32 \mathrm{c}$ as the sole isomer. The structure of tetracycles $32 a-c$ could be confirmed from the X-ray crystal structure of $32 \mathrm{a}^{24}$

Interestingly, the propargylic alcohol of enynes $4 \mathbf{b}, \mathbf{c}$ was substituted by a second molecule of alcohol in the gold(I)catalyzed cyclization process. In order to elucidate the order of events in this transformation, the closely related system 34 in which the 1,3-diene had been reduced to the corresponding alkane was submitted to the optimized reaction conditions for the gold(I)-catalyzed alkoxycyclization (Scheme 8). However, after $2 \mathrm{~h}$ only hydroxyketone 35 and unreacted 34 were detected from the crude mixture and no substitution of the propargylic alcohol was observed. ${ }^{26}$ This result supports a catalytic cycle in which the propargylic alcohol in $4 b, c$ is eliminated after the cyclization of the enyne by the attack of a molecule of methanol to intermediate 36, which generates $\alpha, \beta$ unsaturated gold(I) carbene intermediate $37 .{ }^{27}$ The attack of a second molecule of alcohol to 37 forms 38, which releases tetracycles $\mathbf{3 2}$ by protodeauration (Scheme 9).

Ketoenynes $4 \mathbf{d}$,e were also prepared by direct oxidation of $\mathbf{4 b , c}$, and their alkoxycyclization under the optimized reaction conditions provided mixtures of the two possible regioisomeric products $39 \mathbf{a}^{\prime}, \mathbf{b}^{\prime}$ and $39 \mathbf{a}^{\prime \prime}, \mathbf{b}^{\prime \prime},{ }^{28}$ which could be separated by preparative chromatography (Scheme 10). Water could also be used as the external nucleophile to afford inseparable mixtures of regioisomeric allylic alcohols $39 \mathrm{c}^{\prime}, \mathbf{d}^{\prime} / 39 \mathrm{c}^{\prime \prime}, \mathrm{d}^{\prime \prime}$ in moderate yields.

\section{CONCLUSION}

In summary, the $[3,5,5,7]$ tetracyclic core of echinopines can be readily accessed through the gold(I)-catalyzed alkoxycyclization of tricyclic cyclopropyl-tethered 1,6-enynes bearing an $\mathrm{O}$ functionality at the propargylic position, giving access to functionalized echinopine analogues as single stereoisomers. Furthermore, the cyclization of 1,5-enyne 3a uncovered an unexpected migration of the cyclopropane functionality, thus providing access to the complex natural-product-like $[3,6,5,7]$ tetracycle 12 .

\section{EXPERIMENTAL SECTION}

General Remarks. Chemicals and solvents for chromatography were used as received. Solvents used in reactions under an inert atmosphere were dried by passing through an activated alumina column on a solvent purification system. Analytical thin-layer chromatography was carried out using TLC-aluminum sheets with $0.2 \mathrm{~mm}$ of silica gel (Merck FG254) with UV light as the visualizing agent or an acidic solution of vanillin in ethanol as the developing agent. Purifications by chromatography were carried out using flash grade silica gel (SDS Chromatogel 60 ACC, $40-60 \mathrm{~mm}$ ). Preparative TLC was performed on $20 \mathrm{~cm} \times 20 \mathrm{~cm}$ silica gel plates. Organic solutions were concentrated under reduced pressure on a rotary evaporator. NMR spectra were recorded at $298 \mathrm{~K}$ on 300,400 , and $500 \mathrm{MHz}$ devices. ${ }^{1} \mathrm{H}$ and ${ }^{13} \mathrm{C}$ chemical shifts $(\delta)$ are given in ppm relative to TMS, and coupling constants $(J)$ in $\mathrm{Hz}$. Mass spectra were recorded employing TOF mass analyzers (ESI, APCI). Melting points were determined by observation of the fusion of the solids placed in a capillary, through a magnifying glass. Crystal structure determinations were carried out using a diffractometer equipped with an APPEX $24 \mathrm{~K}$ $\mathrm{CCD}$ area detector, an FR591 rotating anode with Mo K $\alpha$ radiation, Montel mirrors as the monochromator, and a Kryoflex low temperature device $\left(T=-173{ }^{\circ} \mathrm{C}\right)$. Full-sphere data collection was used with $\omega$ and $\varphi$ scans. Programs used: data collection APEX-2, data reduction Bruker Saint V/.60A, and absorption correction SADABS. Structure solution and refinement: crustal structure solution was achieved using direct methods as implemented in SHELXTL and visualized using the program XP. Missing atoms were subsequently located from difference Fourier synthesis and added to the atom list. Least-squares refinement on $F^{2}$ using all measured intensities was carried out using the program SHELXTL. All non-hydrogen atoms were refined including anisotropic displacement parameters.

tert-Butyl((4-methoxybut-2-yn-1-yl)oxy)dimethylsilane (5a). $\mathrm{NaH}(60 \%$ in mineral oil, $1.89 \mathrm{~g}, 47.2 \mathrm{mmol})$ was added to a solution of 4-((tert-butyldimethylsilyl)oxy)but-2-yn-1-ol ${ }^{29}$ (8.60 g, $\left.42.9 \mathrm{mmol}\right)$ 
Scheme 6. Synthesis and Formal Hydration of Tricyclic 1,6-Enyne 4a

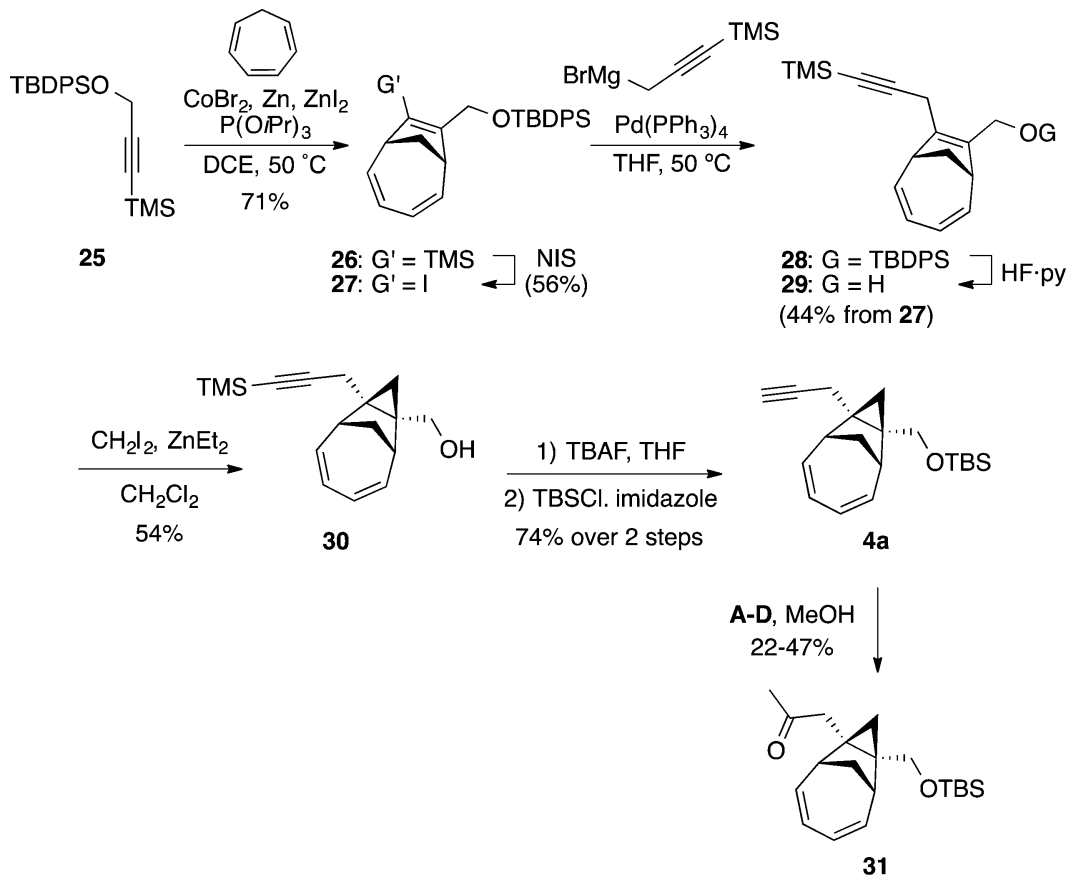

Scheme 7. Synthesis and Alkoxycyclization of $4 b, c$ and CYLview Depiction of the X-ray Crystal Structure of 32a

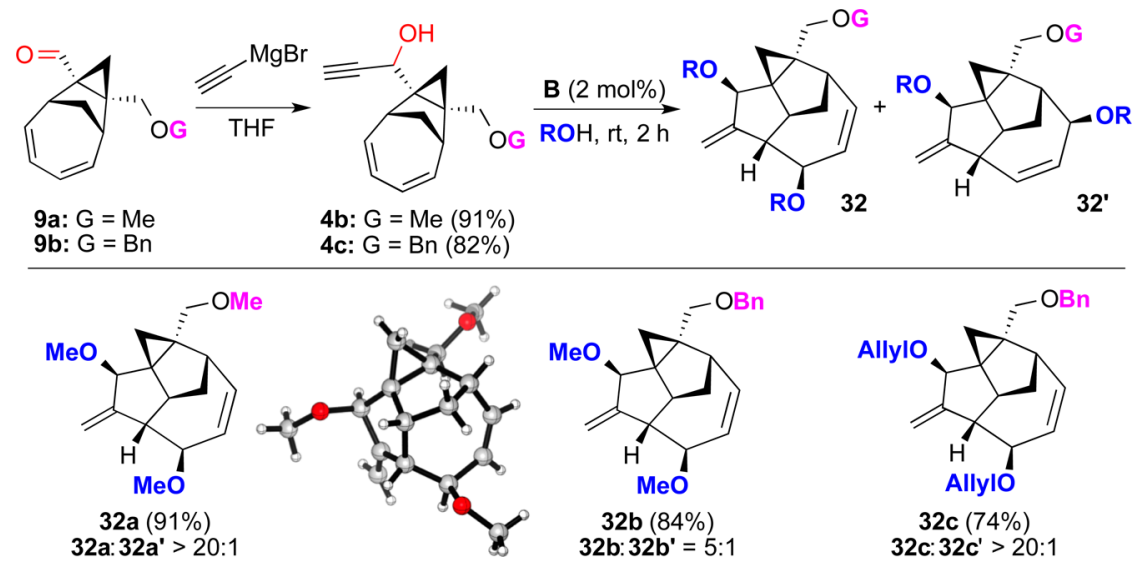

Scheme 8. Synthesis of 34 and Gold(I)-Catalyzed Reaction under the Optimized Alkoxycyclization Reaction Conditions
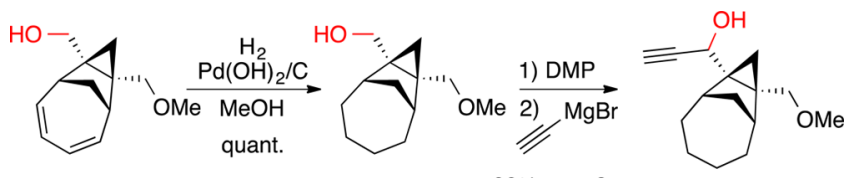

$8 a$

33

$98 \%$ over 2 steps

$$
34
$$$$
34
$$$$
34
$$

35

in anhydrous THF $(210 \mathrm{~mL})$ under argon at $0{ }^{\circ} \mathrm{C}$. The resulting suspension was stirred for $30 \mathrm{~min}$, and then methyl iodide $(3.2 \mathrm{~mL}$, $51.5 \mathrm{mmol}$ ) was slowly added. The reaction mixture was warmed to room temperature and then stirred for $1.5 \mathrm{~h}$. After it was diluted with $\mathrm{Et}_{2} \mathrm{O}(100 \mathrm{~mL})$, the mixture was washed with a saturated solution of $\mathrm{NH}_{4} \mathrm{Cl}(150 \mathrm{~mL})$ and water $(150 \mathrm{~mL})$, the aqueous layers were
Scheme 9. Catalytic Cycle for the Alkoxycyclization of $4 b, c$

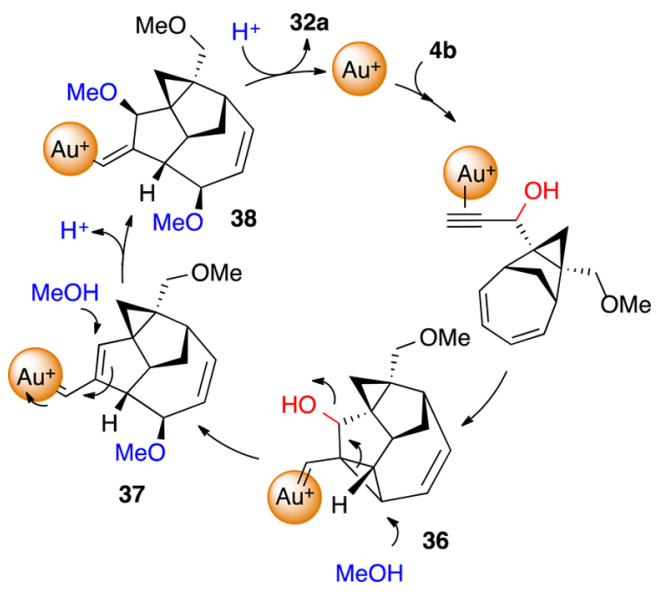

extracted with $\mathrm{Et}_{2} \mathrm{O}(2 \times 100 \mathrm{~mL})$, and the combined organic layers were dried over $\mathrm{MgSO}_{4}$, filtered, and concentrated under reduced pressure. The product was obtained after purification by flash 
Scheme 10. Synthesis and Alkoxycyclization of $4 \mathrm{~d}, \mathrm{e}$
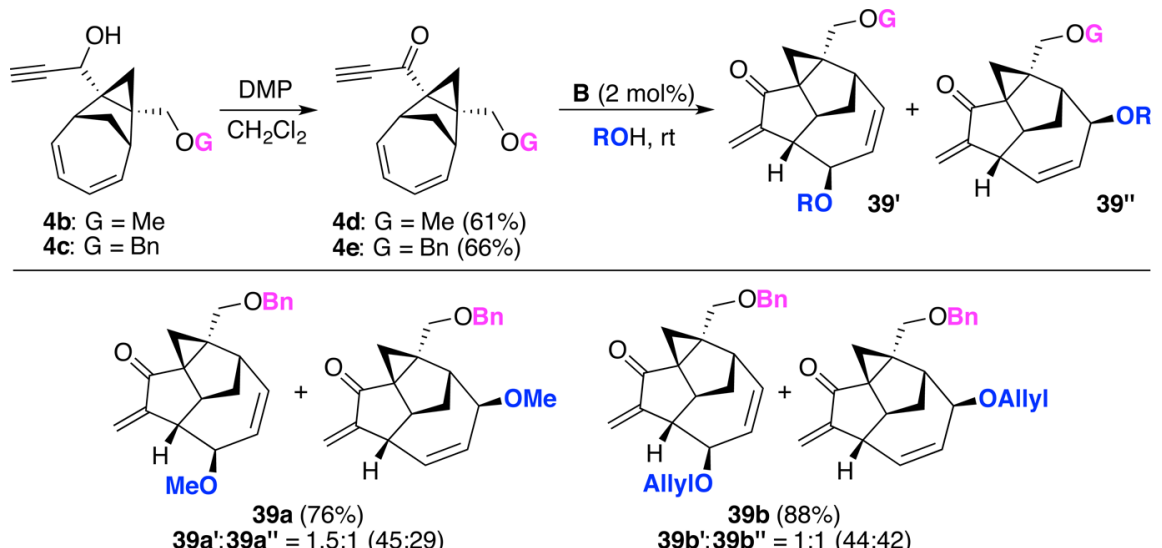

$39 a^{\prime}: 39 a^{\prime \prime}=1.5: 1(45: 29)$

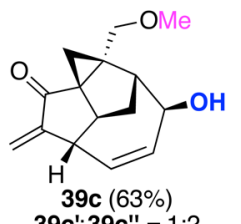

39b': $39 b^{\prime \prime}=1: 1(44: 42)$

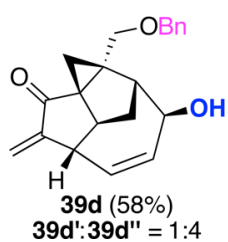

chromatography (cyclohexane/EtOAc $95 / 5)$ as a clear oil ( $8.00 \mathrm{~g}, 37.3$ mmol, yield $87 \%) .{ }^{1} \mathrm{H}$ NMR $\left(400 \mathrm{MHz}, \mathrm{CDCl}_{3}\right): \delta 4.38(\mathrm{t}, J=1.8 \mathrm{~Hz}$, $2 \mathrm{H}), 4.15(\mathrm{t}, J=1.8 \mathrm{~Hz}, 2 \mathrm{H}), 3.40(\mathrm{~s}, 3 \mathrm{H}), 0.93(\mathrm{~s}, 9 \mathrm{H}), 0.14(\mathrm{~s}, 6 \mathrm{H})$. ${ }^{13} \mathrm{C} \mathrm{NMR}\left(101 \mathrm{MHz}, \mathrm{CDCl}_{3}\right): \delta 85.1,80.6,60.0,57.6,51.7,25.8,18.3$, -5.2. HRMS (ESI+): $m / z$ calcd for $\mathrm{C}_{11} \mathrm{H}_{22} \mathrm{NaO}_{2} \mathrm{Si}[\mathrm{M}+\mathrm{Na}]^{+}$, 237.1281; found, 237.1278.

Synthesis of $6 . \mathrm{CoBr}_{2}(306.1 \mathrm{mg}, 1.40 \mathrm{mmol}), \mathrm{Zn}(366.1 \mathrm{mg}, 5.60$ $\mathrm{mmol})$, and $\mathrm{ZnI}_{2}(1.79 \mathrm{~g}, 5.60 \mathrm{mmol})$ were suspended in anhydrous 1,2-dichloroethane $(20 \mathrm{~mL})$ under argon. Then $\mathrm{P}(\mathrm{O} i \mathrm{Pr})_{3}(0.69 \mathrm{~mL}$, $2.80 \mathrm{mmol}$ ) was added, followed by cycloheptatriene $(4.36 \mathrm{~mL}, 41.98$ $\mathrm{mmol})$ and a solution of $5^{30}(27.99 \mathrm{mmol})$ in dry 1,2-dichloroethane $(8 \mathrm{~mL})$. The resulting mixture was stirred at $50{ }^{\circ} \mathrm{C}$ for $16 \mathrm{~h}$ and then filtered through a pad of Celite and concentrated under reduced pressure. Purification of the resulting crude by flash chromatography (cyclohexane/EtOAc $1 / 0$ to 95/5) afforded compounds 6 .

tert-Butyl(((1R*,6S*)-8-(methoxymethyl)bicyclo[4.2.1]nona-2,4,7trien-7-yl)methoxy)dimethylsilane (6a). Pale yellow oil (6.69 g, 21.83 mmol, yield $78 \%) .{ }^{1} \mathrm{H}$ NMR $\left(300 \mathrm{MHz}, \mathrm{CDCl}_{3}\right): \delta 6.29-6.11(\mathrm{~m}$, $2 \mathrm{H}), 5.84-5.74(\mathrm{~m}, 2 \mathrm{H}), 4.35(\mathrm{~d}, J=13.1 \mathrm{~Hz}, 1 \mathrm{H}), 4.23(\mathrm{~d}, J=13.1$ $\mathrm{Hz}, 1 \mathrm{H}), 4.12(\mathrm{~d}, J=12.3 \mathrm{~Hz}, 1 \mathrm{H}), 3.92(\mathrm{~d}, J=12.3 \mathrm{~Hz}, 1 \mathrm{H}), 3.41(\mathrm{t}, J$ $=7.1 \mathrm{~Hz}, 1 \mathrm{H}), 3.32(\mathrm{~d}, J=7.1 \mathrm{~Hz}, 1 \mathrm{H}), 3.27(\mathrm{~s}, 3 \mathrm{H}), 2.29-2.17(\mathrm{~m}$, $1 \mathrm{H}), 1.61(\mathrm{~d}, J=11.6 \mathrm{~Hz}, 1 \mathrm{H}), 0.93(\mathrm{~s}, 9 \mathrm{H}), 0.08(\mathrm{~s}, 3 \mathrm{H}), 0.07(\mathrm{~s}$, $3 \mathrm{H}) .{ }^{13} \mathrm{C}$ NMR (75 MHz, $\left.\mathrm{CDCl}_{3}\right): \delta 139.8,139.8,138.4,132.1,124.3$, $124.2,66.2,57.8,57.2,45.4,44.7,30.0,25.9,18.4,-5.3,-5.4$. HRMS (ESI+): $m / z$ calcd for $\mathrm{C}_{18} \mathrm{H}_{30} \mathrm{NaO}_{2} \mathrm{Si}[\mathrm{M}+\mathrm{Na}]^{+}, 329.1907$; found, 329.1904.

$\left(\left(\left(1 R^{*}, 65 *\right)-8-((\right.\right.$ Benzyloxy)methyl)bicyclo[4.2.1]nona-2,4,7-trien7-yl)methoxy) (tert-butyl)dimethylsilane (6b). Colorless oil (5.89 g, $15.39 \mathrm{mmol}$, yield 55\%). ${ }^{1} \mathrm{H}$ NMR (300 $\left.\mathrm{MHz}, \mathrm{CDCl}_{3}\right): \delta 7.40-7.29$ (m, 5H), 6.26-6.14 (m, 2H), 5.84-5.74 (m, 2H), $4.48(\mathrm{~d}, J=11.8$ $\mathrm{Hz}, 1 \mathrm{H}), 4.41(\mathrm{~d}, J=11.9 \mathrm{~Hz}, 1 \mathrm{H}), 4.32(\mathrm{~d}, J=13.0 \mathrm{~Hz}, 1 \mathrm{H}), 4.20(\mathrm{~d}$, $J=12.6 \mathrm{~Hz}, 2 \mathrm{H}), 4.04(\mathrm{~d}, J=12.4 \mathrm{~Hz}, 1 \mathrm{H}), 3.43(\mathrm{t}, J=7.1 \mathrm{~Hz}, 1 \mathrm{H})$, $3.37(\mathrm{t}, J=7.1 \mathrm{~Hz}, 1 \mathrm{H}), 2.25(\mathrm{dt}, J=12.7,6.7 \mathrm{~Hz}, 1 \mathrm{H}), 1.62(\mathrm{~d}, J=$ $11.4 \mathrm{~Hz}, 1 \mathrm{H}), 0.92(\mathrm{~s}, 9 \mathrm{H}), 0.07(\mathrm{~s}, 3 \mathrm{H}), 0.07(\mathrm{~s}, 3 \mathrm{H}) .{ }^{13} \mathrm{C}$ NMR $(126$ $\mathrm{MHz}_{\mathrm{CDCl}}$ ): $\delta 139.9,139.8,138.5,138.5,132.1,128.3,127.8,127.5$, 124.3, 124.3, 71.7, 63.7, 57.2, 45.5, 44.7, 30.0, 25.9, 18.4, -5.3, -5.4. HRMS (ESI+): $m / z$ calcd for $\mathrm{C}_{24} \mathrm{H}_{34} \mathrm{NaO}_{2} \mathrm{Si}[\mathrm{M}+\mathrm{Na}]^{+}, 405.2220$; found, 405.2230 .

Synthesis of 7. TBAF (1.0 M in THF, $22.8 \mathrm{~mL}, 22.8 \mathrm{mmol}$ ) was added to a solution of $6(11.4 \mathrm{mmol})$ in anhydrous THF $(200 \mathrm{~mL})$ at $0{ }^{\circ} \mathrm{C}$ under argon. The mixture was stirred at room temperature for 2 $\mathrm{h}$ and then diluted with $\mathrm{Et}_{2} \mathrm{O}(100 \mathrm{~mL})$ and washed with saturated solution of $\mathrm{NH}_{4} \mathrm{Cl}(150 \mathrm{~mL})$ and water $(150 \mathrm{~mL})$. The aqueous layers were extracted with $\mathrm{Et}_{2} \mathrm{O}(2 \times 100 \mathrm{~mL})$, and the combined organic

layers were dried over $\mathrm{MgSO}_{4}$, filtered, and concentrated under reduced pressure. Purification by flash chromatography (cyclohexane/ EtOAc 7/3) afforded products 7.

$\left(\left(1 R^{*}, 6 \mathrm{~S} *\right)\right.$-8-(Methoxymethyl)bicyclo[4.2.1]nona-2,4,7-trien-7yl)methanol (7a). Colorless oil (1.62 g, $8.4 \mathrm{mmol}$, yield $74 \%) .{ }^{1} \mathrm{H}$ NMR (400 MHz, $\left.\mathrm{CDCl}_{3}\right): \delta 6.30-6.14(\mathrm{~m}, 2 \mathrm{H}), 5.89-5.78(\mathrm{~m}, 2 \mathrm{H})$, $4.30(\mathrm{dd}, J=13.2,6.3 \mathrm{~Hz}, 1 \mathrm{H}), 4.24(\mathrm{dd}, J=13.2,4.9 \mathrm{~Hz}, 1 \mathrm{H}), 4.17$ $(\mathrm{d}, J=12.6 \mathrm{~Hz}, 1 \mathrm{H}), 4.00(\mathrm{~d}, J=12.4 \mathrm{~Hz}, 1 \mathrm{H}), 3.38-3.26(\mathrm{~m}, 2 \mathrm{H})$, $3.32(\mathrm{~s}, 3 \mathrm{H}), 2.26(\mathrm{dtt}, J=11.5,6.8,1.3 \mathrm{~Hz}, 1 \mathrm{H}), 1.95(\mathrm{t}, J=5.7 \mathrm{~Hz}$, $1 \mathrm{H}), 1.61(\mathrm{~d}, J=11.4 \mathrm{~Hz}, 1 \mathrm{H}) .{ }^{13} \mathrm{C} \operatorname{NMR}\left(101 \mathrm{MHz}, \mathrm{CDCl}_{3}\right): \delta$ 139.3, 139.3, 137.6, 133.3, 124.6, 125,6, 66.6, 58.1, 57.6, 45.7, 45.7, 30.0. HRMS (ESI+): $m / z$ calcd for $\mathrm{C}_{12} \mathrm{H}_{16} \mathrm{NaO}_{2}[\mathrm{M}+\mathrm{Na}]^{+}$, 215.1043; found, 215.1042 .

$\left(\left(1 R^{*}, 6 S^{*}\right)-8-((\right.$ Benzyloxy)methyl)bicyclo[4.2.1]nona-2,4,7-trien-7yl)methanol (7b). Colorless oil $(2.29 \mathrm{~g}, 8.55 \mathrm{mmol}$, yield $75 \%) .{ }^{1} \mathrm{H}$ NMR (500 MHz, $\left.\mathrm{CDCl}_{3}\right): \delta 7.41-7.30(\mathrm{~m}, 5 \mathrm{H}), 6.28-6.16(\mathrm{~m}, 2 \mathrm{H})$, 5.86-5.84 (m, 1H), 5.84-5.81 (m, 1H), $4.52(\mathrm{~d}, J=11.8 \mathrm{~Hz}, 1 \mathrm{H})$, $4.46(\mathrm{~d}, J=11.8 \mathrm{~Hz}, 1 \mathrm{H}), 4.31-4.19(\mathrm{~m}, 3 \mathrm{H}), 4.11(\mathrm{~d}, J=12.5 \mathrm{~Hz}$, $1 \mathrm{H}), 3.35(\mathrm{t}, J=7.0 \mathrm{~Hz}, 2 \mathrm{H}), 2.27(\mathrm{dtt}, J=11.5,6.8,1.2 \mathrm{~Hz}, 1 \mathrm{H}), 1.76$ (s, $1 \mathrm{H}), 1.63(\mathrm{~d}, J=11.4 \mathrm{~Hz}, 1 \mathrm{H}) .{ }^{13} \mathrm{C} \mathrm{NMR}\left(101 \mathrm{MHz}, \mathrm{CDCl}_{3}\right): \delta$ 139.4, 139.4, 138.1, 137.7, 133.5, 128.4, 127.8, 127.7, 124.6, 124.6, 72.2, 63.9, 57.5, 45.7, 45.7, 30.0. HRMS (ESI+): $\mathrm{m} / z$ calcd for $\mathrm{C}_{18} \mathrm{H}_{20} \mathrm{NaO}_{2}[\mathrm{M}+\mathrm{Na}]^{+}$, 291.1356; found, 291.1365.

Synthesis of 8. Diiodomethane $(0.61 \mathrm{~mL}, 7.57 \mathrm{mmol})$ and $\mathrm{ZnEt}_{2}$ (1.0 M in hexanes, $15.75 \mathrm{~mL}, 15.75 \mathrm{mmol}$ ) were added to a solution of $7(6.30 \mathrm{mmol})$ in anhydrous $\mathrm{CH}_{2} \mathrm{Cl}_{2}(210 \mathrm{~mL})$ at $0{ }^{\circ} \mathrm{C}$. The resulting mixture was warmed to room temperature and stirred until TLC analysis showed complete disappearance of the starting material (5-12 $\mathrm{h})$. The reaction mixture was quenched by the slow addition of a saturated aqueous $\mathrm{Na} / \mathrm{K}$-tartrate solution $(100 \mathrm{~mL})$, and after it was stirred for $30 \mathrm{~min}$ the organic layer was separated, the aqueous layer was extracted with $\mathrm{CH}_{2} \mathrm{Cl}_{2}(100 \mathrm{~mL})$, and the combined organic phases were dried over $\mathrm{MgSO}_{4}$ and concentrated under reduced pressure. Purification by flash chromatography (cyclohexane/EtOAc $7 / 3)$ afforded products 8 .

$\left(\left(1 R^{*}, 6 S^{*}, 7 R^{*}, 9 S^{*}\right)-9-\left(\right.\right.$ Methoxymethyl)tricyclo[4.3.1.0 $\left.0^{7,9}\right]$ deca2,4-dien-7-yl)methanol (8a). Colorless oil (1.22 g, $5.92 \mathrm{mmol}$, yield 94\%). ${ }^{1} \mathrm{H}$ NMR (500 MHz, $\left.\mathrm{CDCl}_{3}\right): \delta 6.11-6.00(\mathrm{~m}, 2 \mathrm{H}), 5.85-5.74$ $(\mathrm{m}, 2 \mathrm{H}), 4.01(\mathrm{dd}, J=10.0,1.2 \mathrm{~Hz}, 1 \mathrm{H}), 3.84(\mathrm{~d}, J=11.7 \mathrm{~Hz}, 1 \mathrm{H})$, $3.67(\mathrm{~d}, J=11.7 \mathrm{~Hz}, 1 \mathrm{H}), 3.38(\mathrm{~s}, 3 \mathrm{H}), 3.10(\mathrm{~d}, J=10.0 \mathrm{~Hz}, 1 \mathrm{H})$, $2.86-2.78(\mathrm{~m}, 2 \mathrm{H}), 2.01(\mathrm{dtt}, J=12.8,6.3,1.3 \mathrm{~Hz}, 1 \mathrm{H}), 1.78(\mathrm{~d}, J=$ $13.1 \mathrm{~Hz}, 1 \mathrm{H}), 1.73(\mathrm{~d}, J=13.0 \mathrm{~Hz}, 1 \mathrm{H}), 0.81(\mathrm{~d}, J=5.6 \mathrm{~Hz}, 1 \mathrm{H}), 0.26$ $(\mathrm{d}, J=5.6 \mathrm{~Hz}, 1 \mathrm{H}) .{ }^{13} \mathrm{C}$ NMR $\left(101 \mathrm{MHz}, \mathrm{CDCl}_{3}\right): \delta 137.3,136.5$, $125.9,125.0,73.4,64.2,58.8,42.1,42.0,41.4,39.6,26.6,14.4$. HRMS 
(ESI+): $m / z$ calcd for $\mathrm{C}_{13} \mathrm{H}_{18} \mathrm{NaO}_{2}[\mathrm{M}+\mathrm{Na}]^{+}, 229.1199$; found, 229.1207.

$\left(\left(1 R^{*}, 6 \mathrm{~S} *, 7 R^{*}, 9 \mathrm{~S} *\right)-9-((\right.$ Benzyloxy)methyl)tricyclo[4.3.1.0 7,9$] \mathrm{deca}-$ 2,4-dien-7-yl)methanol (8b). Colorless oil (1.53 g, $5.42 \mathrm{mmol}$, yield 86\%). ${ }^{1} \mathrm{H}$ NMR (500 MHz, $\left.\mathrm{CDCl}_{3}\right): \delta 7.39-7.33(\mathrm{~m}, 4 \mathrm{H}), 7.32-7.29$ $(\mathrm{m}, 1 \mathrm{H}), 6.10-6.04(\mathrm{~m}, 1 \mathrm{H}), 6.01-5.95(\mathrm{~m}, 1 \mathrm{H}), 5.81-5.70(\mathrm{~m}, 2 \mathrm{H})$, $4.59(\mathrm{~d}, J=12.1 \mathrm{~Hz}, 1 \mathrm{H}), 4.52(\mathrm{~d}, J=12.1 \mathrm{~Hz}, 1 \mathrm{H}), 4.08(\mathrm{dd}, J=10.0$, $1.2 \mathrm{~Hz}, 1 \mathrm{H}), 3.82(\mathrm{dd}, J=11.7,7.0 \mathrm{~Hz}, 1 \mathrm{H}), 3.63(\mathrm{~d}, J=11.6 \mathrm{~Hz}, 1 \mathrm{H})$, $3.21(\mathrm{~d}, J=10.0 \mathrm{~Hz}, 1 \mathrm{H}), 2.87(\mathrm{t}, J=6.2 \mathrm{~Hz}, 1 \mathrm{H}), 2.81(\mathrm{t}, J=6.2 \mathrm{~Hz}$, $1 \mathrm{H}), 2.01(\mathrm{dtt}, J=12.8,6.3,1.3 \mathrm{~Hz}, 1 \mathrm{H}), 1.79(\mathrm{~d}, J=13.0 \mathrm{~Hz}, 1 \mathrm{H})$, $1.68(\mathrm{~s}, 1 \mathrm{H}), 0.83(\mathrm{~d}, J=5.6 \mathrm{~Hz}, 1 \mathrm{H}), 0.26(\mathrm{~d}, J=5.6 \mathrm{~Hz}, 1 \mathrm{H}) .{ }^{13} \mathrm{C}$ $\operatorname{NMR}\left(126 \mathrm{MHz}, \mathrm{CDCl}_{3}\right): \delta 138.5,137.3,136.5,128.4,127.7,127.6$, 125.9, 125.0, 72.9, 70.9, 64.3, 42.1, 42.1, 41.5, 39.7, 26.6, 14.6. HRMS (ESI+): $m / z$ calcd for $\mathrm{C}_{19} \mathrm{H}_{22} \mathrm{NaO}_{2}[\mathrm{M}+\mathrm{Na}]^{+}, 305.1512$; found, 305.1517.

Synthesis of 9. Dess-Martin periodinane $(2.67 \mathrm{~g}, 6.30 \mathrm{mmol})$ was added to a solution of $8(4.85 \mathrm{mmol})$ in $\mathrm{CH}_{2} \mathrm{Cl}_{2}(50 \mathrm{~mL})$. After the addition of 1 drop of water the resulting suspension was stirred at room temperature for $1 \mathrm{~h}$ and then washed with a $1 / 1$ mixture of a saturated solution of $\mathrm{Na}_{2} \mathrm{~S}_{2} \mathrm{O}_{3} / \mathrm{Na}_{2} \mathrm{CO}_{3}(40 \mathrm{~mL})$. The organic layer was dried over $\mathrm{MgSO}_{4}$, filtered, and concentrated under reduced pressure. The product was obtained after purification by flash chromatography (cyclohexane/EtOAc 7/3).

$\left(1 R^{*}, 6 S^{*}, 7 R^{*}, 9 S^{*}\right)-9-\left(\right.$ Methoxymethyl)tricyclo[4.3.1.0 $\left.0^{7,9}\right]$ deca-2,4diene-7-carbaldehyde (9a). Colorless oil ( $871.7 \mathrm{mg}, 4.27 \mathrm{mmol}$, yield 88\%). ${ }^{1} \mathrm{H}$ NMR (500 MHz, $\mathrm{CDCl}_{3}$ ): $\delta 9.39$ (s, 1H), 6.31 (ddq, $J=$ $11.4,7.5,1.0 \mathrm{~Hz}, 1 \mathrm{H}), 5.99$ (ddq $J=12.3,7.5,1.0 \mathrm{~Hz}, 1 \mathrm{H}), 5.79$ (ddd, $J=11.4,7.4,0.9 \mathrm{~Hz}, 1 \mathrm{H}), 5.72(\mathrm{ddd}, J=12.3,7.4,0.8 \mathrm{~Hz}, 1 \mathrm{H}), 3.95$ $(\mathrm{dd}, J=9.9,1.4 \mathrm{~Hz}, 1 \mathrm{H}), 3.40(\mathrm{~s}, 3 \mathrm{H}), 3.35(\mathrm{~d}, J=9.9 \mathrm{~Hz}, 1 \mathrm{H}), 3.00-$ $2.95(\mathrm{~m}, 1 \mathrm{H}), 2.88(\mathrm{t}, J=7.0 \mathrm{~Hz}, 1 \mathrm{H}), 2.05(\mathrm{dtt}, J=12.9,6.4,1.3 \mathrm{~Hz}$, $1 \mathrm{H}), 1.91(\mathrm{~d}, J=13.2 \mathrm{~Hz}, 1 \mathrm{H}), 1.25(\mathrm{~d}, J=5.8 \mathrm{~Hz}, 1 \mathrm{H}), 1.19(\mathrm{dd}, J=$ $5.8,1.6 \mathrm{~Hz}, 1 \mathrm{H}) .{ }^{13} \mathrm{C} \mathrm{NMR}\left(126 \mathrm{MHz}, \mathrm{CDCl}_{3}\right): \delta 200.0,136.6,134.8$, $125.7,124.5,71.5,58.9,51.8,46.0,41.3,39.4,26.5,17.5$. HRMS (ESI $+): m / z$ calcd for $\mathrm{C}_{13} \mathrm{H}_{16} \mathrm{NaO}_{2}[\mathrm{M}+\mathrm{Na}]^{+}, 227.1043$; found, 227.1040.

$\left(1 R^{*}, 6 S^{*}, 7 R^{*}, 9 S^{*}\right)-9-\left(\left(\right.\right.$ Benzyloxy)methyl)tricyclo[4.3.1.0 $\left.0^{7,9}\right]$ deca2,4-diene-7-carbaldehyde (9b). Colorless oil (1.06 g, $3.78 \mathrm{mmol}$, yield $78 \%) .{ }^{1} \mathrm{H}$ NMR $\left(500 \mathrm{MHz}, \mathrm{CDCl}_{3}\right): \delta 9.36(\mathrm{~s}, 1 \mathrm{H}), 7.42-7.34$ (m, 4H), 7.34-7.29 (m, 1H), 6.33-6.27 (m, 1H), 5.97-5.89 (m, 1H), $5.78-5.67(\mathrm{~m}, 2 \mathrm{H}), 4.60(\mathrm{~d}, J=11.9 \mathrm{~Hz}, 1 \mathrm{H}), 4.52(\mathrm{~d}, J=11.9 \mathrm{~Hz}$, $1 \mathrm{H}), 4.04(\mathrm{dd}, J=9.9,1.3 \mathrm{~Hz}, 1 \mathrm{H}), 3.48(\mathrm{~d}, J=9.8 \mathrm{~Hz}, 1 \mathrm{H}), 2.97(\mathrm{dd}$, $J=7.5,6.3 \mathrm{~Hz}, 1 \mathrm{H}), 2.93(\mathrm{t}, J=7.0 \mathrm{~Hz}, 1 \mathrm{H}), 2.06(\mathrm{dtt}, J=12.9,6.4$, $1.3 \mathrm{~Hz}, 1 \mathrm{H}), 1.91(\mathrm{~d}, J=13.2 \mathrm{~Hz}, 1 \mathrm{H}), 1.26(\mathrm{~d}, J=5.8 \mathrm{~Hz}, 1 \mathrm{H}), 1.21$ $(\mathrm{dd}, J=5.9,1.3 \mathrm{~Hz}, 1 \mathrm{H}) .{ }^{13} \mathrm{C}$ NMR $\left(126 \mathrm{MHz}, \mathrm{CDCl}_{3}\right): \delta 199.9$, $138.2,136.6,134.8,128.4,127.7,125.7,124.4,73.2,69.2,51.9,46.1$, 41.4, 39.4, 26.4, 17.7. HRMS (ESI+): $m / z$ calcd for $\mathrm{C}_{19} \mathrm{H}_{20} \mathrm{NaO}_{2}[\mathrm{M}+$ $\mathrm{Na}]^{+}, 303.1356$; found, 303.1361.

$\left(1 R^{*}, 6 S^{*}, 7 S^{*}, 9 S^{*}\right)-7-$ Ethynyl-9-(methoxymethyl)tricyclo[4.3.1.0 $0^{7,9}$ ] deca-2,4-diene (3a). Ohira-Bestmann reagent $(790 \mathrm{mg}$, $4.98 \mathrm{mmol}$ ) and $\mathrm{K}_{2} \mathrm{CO}_{3}(947.3 \mathrm{mg}, 6.85 \mathrm{mmol})$ were sequentially added to a solution of $9 \mathrm{a}(700 \mathrm{mg}, 3.43 \mathrm{mmol})$ in $\mathrm{MeOH}(34 \mathrm{~mL})$, and the resulting mixture was stirred at room temperature for $16 \mathrm{~h}$. Then the volatiles were removed under reduced pressure and the residue was dissolved in EtOAc $(30 \mathrm{~mL})$ and washed with water $(30$ $\mathrm{mL})$ and brine $(30 \mathrm{~mL})$. The organic layer was dried over $\mathrm{MgSO}_{4}$, filtered, and concentrated under reduced pressure. Purification by flash chromatography (cyclohexane/EtOAc 85/15) afforded the product as a colorless oil (391.6 mg, $1.96 \mathrm{mmol}$, yield 57\%). ${ }^{1} \mathrm{H}$ NMR (500 MHz, $\left.\mathrm{CDCl}_{3}\right): \delta 6.09-5.96(\mathrm{~m}, 2 \mathrm{H}), 5.85-5.78(\mathrm{~m}, 2 \mathrm{H}), 4.09(\mathrm{dd}, J=10.2$, $1.6 \mathrm{~Hz}, 1 \mathrm{H}), 3.40(\mathrm{~s}, 3 \mathrm{H}), 3.10(\mathrm{~d}, J=10.2 \mathrm{~Hz}, 1 \mathrm{H}), 2.82(\mathrm{dd}, J=6.7$, $5.2 \mathrm{~Hz}, 1 \mathrm{H}), 2.80(\mathrm{dd}, J=6.4,5.3 \mathrm{~Hz}, 1 \mathrm{H}), 2.00-1.92(\mathrm{~m}, 1 \mathrm{H}), 1.98$ $(\mathrm{s}, 1 \mathrm{H}), 1.85(\mathrm{dd}, J=13.2,0.6 \mathrm{~Hz}, 1 \mathrm{H}), 0.98(\mathrm{dd}, J=5.7,1.6 \mathrm{~Hz}, 1 \mathrm{H})$, $0.57(\mathrm{~d}, J=5.6 \mathrm{~Hz}, 1 \mathrm{H}) .{ }^{13} \mathrm{C} \mathrm{NMR}\left(126 \mathrm{MHz}, \mathrm{CDCl}_{3}\right): \delta 136.5$, 135.6, 125.6, 125.0, 84.5, 74.2, 67.3, 58.8, 42.3, 40.6, 40.5, 30.0, 26.7, 17.2. HRMS (APCI+): $m / z$ calcd for $\mathrm{C}_{14} \mathrm{H}_{17} \mathrm{O}[\mathrm{M}+\mathrm{H}]^{+}, 201.1274$; found, 201.1271.

1-((1 $\left.R^{*}, 6 S^{*}, 7 R^{*}, 9 S^{*}\right)-9$-(Methoxymethyl)tricyclo[4.3.1.0 $\left.{ }^{7,9}\right]$ deca-2,4-dien-7-yl)ethan-1-one (10). To a solution of 3a (20.0 $\mathrm{mg}, 0.1 \mathrm{mmol})$ in $\mathrm{MeOH}(1 \mathrm{~mL})$ was added gold(I) complex $\mathbf{B}^{31}$ (7.9 $\mathrm{mg}, 0.005 \mathrm{mmol})$, and the resulting mixture was stirred at room temperature for $2 \mathrm{~h}$. The reaction was quenched by the addition of 1 drop of $\mathrm{Et}_{3} \mathrm{~N}$, and the volatiles were removed under reduced pressure. Purification by column chromatography (cyclohexane/EtOAc 8/2) afforded the product as a colorless oil $(21.6 \mathrm{mg}, 0.099 \mathrm{mmol}$, yield 99\%). ${ }^{1} \mathrm{H}$ NMR (400 MHz, $\mathrm{CDCl}_{3}$ ): $\delta 6.12-6.02(\mathrm{~m}, 2 \mathrm{H}), 5.82-5.70$ $(\mathrm{m}, 2 \mathrm{H}), 3.96(\mathrm{dd}, J=10.2,1.4 \mathrm{~Hz}, 1 \mathrm{H}), 3.66(\mathrm{~d}, J=10.2 \mathrm{~Hz}, 1 \mathrm{H})$, $3.40(\mathrm{~s}, 3 \mathrm{H}), 3.03(\mathrm{dd}, J=7.4,6.5 \mathrm{~Hz}, 1 \mathrm{H}), 2.86(\mathrm{dd}, J=7.4,6.4 \mathrm{~Hz}$, $1 \mathrm{H}), 2.29$ (s, 3H), 2.05 (dddd, $J=13.0,6.5,5.1,1.4 \mathrm{~Hz}, 1 \mathrm{H}), 1.82(\mathrm{~d}, J$ $=13.2 \mathrm{~Hz}, 1 \mathrm{H}), 1.11(\mathrm{dd}, J=5.0,1.4 \mathrm{~Hz}, 1 \mathrm{H}), 0.83(\mathrm{~d}, J=5.0 \mathrm{~Hz}$, $1 \mathrm{H}) .{ }^{13} \mathrm{C}$ NMR $\left(101 \mathrm{MHz}, \mathrm{CDCl}_{3}\right): \delta 207.9,136.5,135.5,124.9$, 124.9, 72.1, 58.8, 49.4, 44.3, 41.3, 40.9, 31.5, 25.9, 20.8. HRMS (ESI+): $m / z$ calcd for $\mathrm{C}_{14} \mathrm{H}_{18} \mathrm{NaO}_{2}[\mathrm{M}+\mathrm{Na}]^{+}, 241.1199$; found, 241.1193 .

Isolation of the Acetal Intermediate $(1 R *, 6 S *, 7 R *, 95 *)-7-(1,1$ Dimethoxyethyl)-9-(methoxymethyl)tricyclo[4.3.1.0, 7 deca-2,4diene (11). Inside a glovebox, $3 \mathrm{a}(20 \mathrm{mg}, 0.1 \mathrm{mmol})$ was dissolved in anhydrous and degassed $\mathrm{CH}_{2} \mathrm{Cl}_{2}(1 \mathrm{~mL})$ and then anhydrous $\mathrm{MeOH}$ $(41 \mu \mathrm{L}, 1 \mathrm{mmol})$ and gold(I) complex B $(7.9 \mathrm{mg}, 0.005 \mathrm{mmol})$ were sequentially added. The resulting mixture was stirred at room temperature for $30 \mathrm{~min}$ and then quenched by the addition of 1 drop of $\mathrm{Et}_{3} \mathrm{~N}$. After removal of the volatiles under reduced pressure, filtration through a pad of basic $\mathrm{Al}_{2} \mathrm{O}_{3}$ afforded the product as an unstable colorless oil. ${ }^{1} \mathrm{H}$ NMR $\left(300 \mathrm{MHz}, \mathrm{CDCl}_{3}\right): \delta 6.10-5.99(\mathrm{~m}$, $2 \mathrm{H}), 5.80-5.64(\mathrm{~m}, 2 \mathrm{H}), 3.89(\mathrm{dd}, J=9.6,1.4 \mathrm{~Hz}, 1 \mathrm{H}), 3.40(\mathrm{~s}, 3 \mathrm{H})$, $3.36(\mathrm{~d}, J=9.5 \mathrm{~Hz}, 1 \mathrm{H}), 3.20(\mathrm{~s}, 3 \mathrm{H}), 3.04(\mathrm{~s}, 3 \mathrm{H}), 2.83(\mathrm{t}, J=6.7 \mathrm{~Hz}$, $1 \mathrm{H}), 2.73(\mathrm{dd}, J=7.7,6.6 \mathrm{~Hz}, 1 \mathrm{H}), 2.06(\mathrm{dt}, J=12.8,6.4 \mathrm{~Hz}, 1 \mathrm{H})$, $1.75(\mathrm{~d}, J=13.0 \mathrm{~Hz}, 1 \mathrm{H}), 1.40(\mathrm{~s}, 3 \mathrm{H}), 0.80(\mathrm{~d}, J=5.1 \mathrm{~Hz}, 1 \mathrm{H}), 0.63$ $(\mathrm{dd}, J=5.1,1.3 \mathrm{~Hz}, 1 \mathrm{H}) .{ }^{13} \mathrm{C}$ NMR $\left(126 \mathrm{MHz}, \mathrm{CDCl}_{3}\right): \delta 138.4$, 136.5, 125.4, 124.4, 100.7, 72.5, 58.8, 48.8, 48.0, 42.3, 42.1, 29.7, 27.4, 23.2, 17.6, 12.1. HRMS could not be obtained due to decomposition to 10 .

$\left(1 R^{*}, 1 \mathrm{a} R^{*}, 3 R^{*}, 5 \mathrm{a} R^{*}\right)-1$-(Methoxymethyl)-1, 1a,2,2a,5a,7ahexahydro-3H-1,3-methanocyclopropa[f]azulen-3-yl Acetate (12). Gold(I) complex A $(38.6 \mathrm{mg}, 0.05 \mathrm{mmol})$ was added to a solution of $3 \mathrm{a}(200.3 \mathrm{mg}, 1 \mathrm{mmol})$ and $\mathrm{AcOH}(0.57 \mathrm{~mL}, 10 \mathrm{mmol})$ in $\mathrm{CH}_{2} \mathrm{Cl}_{2}(10 \mathrm{~mL})$, and the resulting mixture was stirred at room temperature for $1 \mathrm{~h}$. The reaction was quenched by the addition of 1 drop of $\mathrm{Et}_{3} \mathrm{~N}$, and the volatiles were removed under reduced pressure. Purification by column chromatography (cyclohexane/EtOAc $95 / 5$ to $8 / 2)$ afforded the product as a colorless oil $(158.8 \mathrm{mg}, 0.61 \mathrm{mmol}, \mathrm{dr}=$ $5: 1$, yield $61 \%$ ). Data for the major isomer are as follows. ${ }^{1} \mathrm{H}$ NMR $\left(400 \mathrm{MHz}, \mathrm{CDCl}_{3}\right): \delta 6.53(\mathrm{dt}, J=6.0,0.9 \mathrm{~Hz}, 1 \mathrm{H}), 5.74(\mathrm{dd}, J=5.9$, $3.1 \mathrm{~Hz}, 1 \mathrm{H}), 5.62(\mathrm{ddd}, J=12.5,7.9,2.0 \mathrm{~Hz}, 1 \mathrm{H}), 5.28(\mathrm{ddd}, J=12.4$, 4.3, $1.4 \mathrm{~Hz}, 1 \mathrm{H}), 3.32(\mathrm{~s}, 3 \mathrm{H}), 3.29(\mathrm{~d}, J=10.0 \mathrm{~Hz}, 1 \mathrm{H}), 3.12(\mathrm{~d}, J=$ $10.0 \mathrm{~Hz}, 1 \mathrm{H}), 3.03-2.97(\mathrm{~m}, 1 \mathrm{H}), 2.70(\mathrm{dd}, J=16.8,1.5 \mathrm{~Hz}, 1 \mathrm{H})$, $2.48-2.42(\mathrm{~m}, 1 \mathrm{H}), 2.33(\mathrm{ddd}, J=14.5,7.4,5.1 \mathrm{~Hz}, 1 \mathrm{H}), 2.06(\mathrm{~s}, 3 \mathrm{H})$, $2.05(\mathrm{~d}, J=14.6 \mathrm{~Hz}, 1 \mathrm{H}), 1.65(\mathrm{~d}, J=11.2 \mathrm{~Hz}, 1 \mathrm{H}), 1.39-1.34(\mathrm{~m}$, $1 \mathrm{H}), 1.19(\mathrm{t}, J=8.5 \mathrm{~Hz}, 1 \mathrm{H}) .{ }^{13} \mathrm{C} \mathrm{NMR}\left(101 \mathrm{MHz}, \mathrm{CDCl}_{3}\right): \delta 170.6$, 137.6, 133.4, 126.8, 123.5, 85.9, 81.4, 58.1, 47.4, 42.8, 31.7, 28.1, 24.5, 22.1, 21.7, 19.1. HRMS (ESI+): $m / z$ calcd for $\mathrm{C}_{16} \mathrm{H}_{20} \mathrm{NaO}_{3}[\mathrm{M}+$ $\mathrm{Na}]^{+}, 283.1305$; found, 283.1307 .

$\left(1 R^{*}, 1 \mathrm{a} R^{*}, 3 R^{*}, 5 \mathrm{a} R^{*}\right)-1$-(Methoxymethyl)-1, $1 \mathrm{a}, 2,2 \mathrm{a}, 5 \mathrm{a}, 7 \mathrm{a}-$ hexahydro-3H-1,3-methanocyclopropa[f]azulen-3-ol (18). $\mathrm{K}_{2} \mathrm{CO}_{3}(253.9 \mathrm{mg}, 1.92 \mathrm{mmol})$ was added to a solution of $12(100$ $\mathrm{mg}, 0.38 \mathrm{mmol})$ in a $1 / 1$ mixture of $\mathrm{CH}_{2} \mathrm{Cl}_{2}$ and $\mathrm{MeOH}(4 \mathrm{~mL})$, and the resulting mixture was stirred at $50{ }^{\circ} \mathrm{C}$ for $16 \mathrm{~h}$. After the mixture was cooled to room temperature, the volatiles were removed under reduced pressure and the resulting crude was purified by column chromatography (cyclohexane/EtOAc $7 / 3$ to $1 / 1$ ) to give the product as a colorless oil $(69.7 \mathrm{mg}, 0.32 \mathrm{mmol}, \mathrm{dr}>20: 1$, yield $84 \%) .{ }^{1} \mathrm{H}$ NMR $\left(400 \mathrm{MHz}, \mathrm{CDCl}_{3}\right): \delta 6.00(\mathrm{dt}, J=5.8,0.9 \mathrm{~Hz}, 1 \mathrm{H}), 5.68(\mathrm{dd}, J=5.8$, $3.2 \mathrm{~Hz}, 1 \mathrm{H}$ ), 5.61 (ddd, $J=12.5,7.9,2.0 \mathrm{~Hz}, 1 \mathrm{H}), 5.29$ (ddd, $J=12.4$, $4.3,1.4 \mathrm{~Hz}, 1 \mathrm{H}), 3.35(\mathrm{~s}, 3 \mathrm{H}), 3.31(\mathrm{~d}, J=9.8 \mathrm{~Hz}, 1 \mathrm{H}), 3.15(\mathrm{~d}, J=9.8$ $\mathrm{Hz}, 1 \mathrm{H}), 3.06-2.97(\mathrm{~m}, 1 \mathrm{H}), 2.37$ (ddd, $J=14.5,7.4,5.2 \mathrm{~Hz}, 1 \mathrm{H})$, $2.19(\mathrm{dd}, J=15.9,1.5 \mathrm{~Hz}, 1 \mathrm{H}), 2.11(\mathrm{t}, J=7.1 \mathrm{~Hz}, 1 \mathrm{H}), 2.02(\mathrm{~d}, J=$ $14.6 \mathrm{~Hz}, 1 \mathrm{H}), 1.78(\mathrm{~s}, 1 \mathrm{H}), 1.68(\mathrm{~d}, J=15.9 \mathrm{~Hz}, 1 \mathrm{H}), 1.36(\mathrm{ddd}, J=$ 9.0, 5.4, $1.3 \mathrm{~Hz}, 1 \mathrm{H}), 1.19(\mathrm{t}, J=8.4 \mathrm{~Hz}, 1 \mathrm{H}) .{ }^{13} \mathrm{C}$ NMR $(101 \mathrm{MHz}$, $\left.\mathrm{CDCl}_{3}\right): \delta 141.4,132.6,127.1,123.3,81.8,77.9,58.4,48.8,45.7,35.0$, $28.3,24.6,21.7,18.9$. HRMS (ESI +$): m / z$ calcd for $\mathrm{C}_{14} \mathrm{H}_{18} \mathrm{NaO}_{2}[\mathrm{M}+$ $\mathrm{Na}]^{+}, 241.1199$; found, 241.1205.

$\left(1 R^{*}, 1 \mathrm{a} R^{*}, 3 R^{*}, 5 \mathrm{a} R^{*}\right)-1$-(Methoxymethyl)-1, $1 \mathrm{a}, 2,2 \mathrm{a}, 5 \mathrm{a}, 7 \mathrm{a}-$ hexahydro-3H-1,3-methanocyclopropa[f]azulen-3-yl 4-Nitro- 
benzoate (19). 4-Nitrobenzoyl chloride ( $30.6 \mathrm{mg}, 0.16 \mathrm{mmol}$ ) was added to a solution of $18(30 \mathrm{mg}, 0.14 \mathrm{mmol}), \mathrm{Et}_{3} \mathrm{~N}(38 \mu \mathrm{L}, 0.27$ $\mathrm{mmol})$, and DMAP $(1.2 \mathrm{mg}, 0.01 \mathrm{mmol})$ in anhydrous $\mathrm{CH}_{2} \mathrm{Cl}_{2}(3$ $\mathrm{mL}$ ), and the mixture was stirred at room temperature for $1 \mathrm{~h}$ and then washed with $\mathrm{H}_{2} \mathrm{O}(2 \times 5 \mathrm{~mL})$. The organic layer was dried over $\mathrm{MgSO}_{4}$, filtered, and concentrated under reduced pressure. Purification by column chromatography (cyclohexane/EtOAc 9/1) afforded the product as a white solid $(51.3 \mathrm{mg}, 0.14 \mathrm{mmol}$, yield quantitative). Mp: $136-138{ }^{\circ} \mathrm{C} .{ }^{1} \mathrm{H}$ NMR $\left(500 \mathrm{MHz}, \mathrm{CDCl}_{3}\right): \delta 8.32-8.29(\mathrm{~m}$, $2 \mathrm{H}), 8.24-8.20(\mathrm{~m}, 2 \mathrm{H}), 6.63(\mathrm{dt}, J=5.9,0.9 \mathrm{~Hz}, 1 \mathrm{H}), 5.84(\mathrm{dd}, J=$ $5.9,3.1 \mathrm{~Hz}, 1 \mathrm{H}), 5.68(\mathrm{ddd}, J=12.5,8.0,2.1 \mathrm{~Hz}, 1 \mathrm{H}), 5.34(\mathrm{ddd}, J=$ $12.4,4.2,1.1 \mathrm{~Hz}, 1 \mathrm{H}), 3.32(\mathrm{~d}, J=10.0 \mathrm{~Hz}, 1 \mathrm{H}), 3.25(\mathrm{~s}, 3 \mathrm{H}), 3.12-$ $3.09(\mathrm{~m}, 1 \mathrm{H}), 3.11(\mathrm{~d}, J=10.0 \mathrm{~Hz}, 1 \mathrm{H}), 2.84(\mathrm{dd}, J=16.9,1.6 \mathrm{~Hz}$, $1 \mathrm{H}), 2.73-2.68(\mathrm{~m}, 1 \mathrm{H}), 2.43$ (ddd, $J=14.7,7.4,5.2 \mathrm{~Hz}, 1 \mathrm{H}), 2.16$ $(\mathrm{d}, J=14.7 \mathrm{~Hz}, 1 \mathrm{H}), 1.80(\mathrm{~d}, J=16.8 \mathrm{~Hz}, 1 \mathrm{H}), 1.44(\mathrm{dd}, J=8.9,5.2$ $\mathrm{Hz}, 1 \mathrm{H}), 1.29-1.23(\mathrm{~m}, 1 \mathrm{H}) .{ }^{13} \mathrm{C}$ NMR $\left(126 \mathrm{MHz}, \mathrm{CDCl}_{3}\right): \delta 163.9$, $150.4,137.0,136.9,134.0,130.6,126.7,123.7,123.5,88.0,81.3,58.2$, 47.5, 42.7, 32.0, 28.1, 24.5, 21.7, 19.3. HRMS (ESI + ): $\mathrm{m} / \mathrm{z}$ calcd for $\mathrm{C}_{21} \mathrm{H}_{21} \mathrm{NNaO}_{5}[\mathrm{M}+\mathrm{Na}]^{+}$, 390.1312; found, 390.1315.

$\left(1 R^{*}, 1 \mathrm{a} R^{*}, 3 S^{*}, 5 \mathrm{a} S^{*}\right)-1$-(Methoxymethyl)-1,1a,2,2a,4,5,5a,7aoctahydro-3H-1,3-methanocyclopropa[f]azulen-3-ol (20). A round-bottom flask containing a solution of $18(50 \mathrm{mg}, 0.23 \mathrm{mmol})$ and Crabtree's catalyst $(3.7 \mathrm{mg}, 0.0046 \mathrm{mmol})$ in anhydrous $\mathrm{MeOH}$ ( $3 \mathrm{~mL}$ ) was evacuated and back-filled with $\mathrm{H}_{2}$ (repeated three times). The resulting mixture was stirred at room temperature for $1 \mathrm{~h}$, then the volatiles were removed under reduced pressure, and the crude waqs purified by column chromatography (cyclohexane/EtOAc 1/1) to afford the product as a yellow oil $(46.0 \mathrm{mg}, 0.21 \mathrm{mmol}$, yield $91 \%)$. ${ }^{1} \mathrm{H}$ NMR $\left(400 \mathrm{MHz}, \mathrm{CDCl}_{3}\right): \delta 5.47-5.36(\mathrm{~m}, 2 \mathrm{H}), 3.41-3.33(\mathrm{~m}$, $1 \mathrm{H}), 3.37(\mathrm{~s}, 3 \mathrm{H}), 2.99(\mathrm{~d}, J=9.3 \mathrm{~Hz}, 1 \mathrm{H}), 2.54-2.46(\mathrm{~m}, 1 \mathrm{H}), 2.29-$ $2.20(\mathrm{~m}, 1 \mathrm{H}), 2.06-1.96(\mathrm{~m}, 1 \mathrm{H}), 1.95-1.85(\mathrm{~m}, 4 \mathrm{H}), 1.77-1.58(\mathrm{~m}$, $4 \mathrm{H}), 1.38-1.24(\mathrm{~m}, 2 \mathrm{H}) .{ }^{13} \mathrm{C}$ NMR $\left(101 \mathrm{MHz} \mathrm{CDCl}_{3}\right): \delta$ 135.6, 120.0, 82.0, 74.6, 58.7, 43.5, 40.1, 38.6, 31.5, 29.3, 24.2, 22.4, 21.4, 18.4. HRMS (ESI+): $m / z$ calcd for $\mathrm{C}_{14} \mathrm{H}_{20} \mathrm{NaO}_{2}[\mathrm{M}+\mathrm{Na}]^{+}$, 243.1356; found, 243.1355 .

$\left(1 R^{*}, 1 \mathrm{a} R^{*}, 5 \mathrm{a} S^{*}\right)-1$-(Methoxymethyl)-1a,2,2a,5,5a,7a-hexahydro-1 $\mathrm{H}$-1,3-methanocyclopropa[f] azulene (23). $\mathrm{Tf}_{2} \mathrm{O}(37 \mu \mathrm{L}$, $0.22 \mathrm{mmol}$ ) was slowly added to a solution of $20(40 \mathrm{mg}, 0.18 \mathrm{mmol})$, pyridine $(29 \mu \mathrm{L}, 0.36 \mathrm{mmol})$, and DMAP $(2.2 \mathrm{mg}, 0.018 \mathrm{mmol})$ in anhydrous $\mathrm{CH}_{2} \mathrm{Cl}_{2}(2 \mathrm{~mL})$ at $0{ }^{\circ} \mathrm{C}$. The reaction mixture was warmed to room temperature and after stirring for $1 \mathrm{~h}$ was quenched by the addition of $\mathrm{H}_{2} \mathrm{O}(2 \mathrm{~mL})$. The organic layer was separated, dried over $\mathrm{MgSO}_{4}$, filtered, and concentrated under reduced pressure. Purification by column chromatography (cyclohexane/EtOAc 98/2) afforded the product as a yellow oil (35.6 mg, $0.18 \mathrm{mmol}$, yield $98 \%) .{ }^{1} \mathrm{H}$ NMR $\left(400 \mathrm{MHz}, \mathrm{CDCl}_{3}\right): \delta 5.51$ (bs, $\left.1 \mathrm{H}\right), 5.44$ (ddd, $J=12.4,7.3,1.7 \mathrm{~Hz}$, $1 \mathrm{H}), 5.40-5.34(\mathrm{~m}, 1 \mathrm{H}), 3.63(\mathrm{~d}, J=9.9 \mathrm{~Hz}, 1 \mathrm{H}), 3.40(\mathrm{~s}, 3 \mathrm{H}), 3.28$ $(\mathrm{d}, J=9.9 \mathrm{~Hz}, 1 \mathrm{H}), 2.55-2.49(\mathrm{~m}, 1 \mathrm{H}), 2.27-2.11(\mathrm{~m}, 5 \mathrm{H}), 2.05-$ $2.00(\mathrm{~m}, 1 \mathrm{H}), 1.77-1.73(\mathrm{~m}, 1 \mathrm{H}), 1.72-1.65(\mathrm{~m}, 1 \mathrm{H}), 1.56(\mathrm{t}, J=8.1$, $7.6 \mathrm{~Hz}, 1 \mathrm{H}) .{ }^{13} \mathrm{C}$ NMR $\left(101 \mathrm{MHz}, \mathrm{CDCl}_{3}\right): \delta 147.9,134.1,121.3$, $118.1,79.6,58.6,44.1,38.5,36.7,33.8,30.8,27.9,27.8,21.0$. HRMS (ESI+): $m / z$ calcd for $\mathrm{C}_{14} \mathrm{H}_{18} \mathrm{NaO}[\mathrm{M}+\mathrm{Na}]^{+}, 225.1250$; found, 225.1243 .

tert-Butyldiphenyl(((1R*,6S*)-8-(trimethylsilyl)bicyclo[4.2.1]nona-2,4,7-trien-7-yl)methoxy)silane (26). $\mathrm{CoBr}_{2}(238.6 \mathrm{mg}, 1.09$ $\mathrm{mmol}), \mathrm{Zn}$ (285.4 mg, $4.36 \mathrm{mmol})$, and $\mathrm{ZnI}_{2}(1.39 \mathrm{~g}, 4.36 \mathrm{mmol})$ were suspended in anhydrous 1,2-dichloroethane $(35 \mathrm{~mL})$ under argon. Then $\mathrm{P}\left(\mathrm{O}^{i} \mathrm{Pr}\right)_{3}(0.54 \mathrm{~mL}, 2.18 \mathrm{mmol})$ was added, followed by cycloheptatriene $(3.40 \mathrm{~mL}, 32.73 \mathrm{mmol})$ and a solution of $25^{32}(8.00$ $\mathrm{g}, 21.82 \mathrm{mmol})$ in anhydrous 1,2-dichloroethane $(9 \mathrm{~mL})$. The resulting mixture was stirred at $50{ }^{\circ} \mathrm{C}$ for $30 \mathrm{~h}$ and then filtered through a short pad of silica gel and concentrated under reduced pressure. Purification by flash chromatography (cyclohexane/EtOAc $1 / 0$ to $95 / 5)$ afforded the product as a colorless oil $(7.10 \mathrm{~g}, 15.49$ mmol, yield 71\%). ${ }^{1} \mathrm{H}$ NMR (500 $\left.\mathrm{MHz}, \mathrm{CDCl}_{3}\right): \delta 7.74-7.68(\mathrm{~m}$, $4 \mathrm{H}), 7.48-7.39(\mathrm{~m}, 6 \mathrm{H}), 6.16-6.10(\mathrm{~m}, 1 \mathrm{H}), 6.09-6.03(\mathrm{~m}, 1 \mathrm{H})$, $5.83-5.72(\mathrm{~m}, 2 \mathrm{H}), 4.43(\mathrm{~d}, J=12.8 \mathrm{~Hz}, 1 \mathrm{H}), 4.23(\mathrm{~d}, J=12.7 \mathrm{~Hz}$, $1 \mathrm{H}), 3.69(\mathrm{t}, J=7.2 \mathrm{~Hz}, 1 \mathrm{H}), 3.14(\mathrm{t}, J=7.0 \mathrm{~Hz}, 1 \mathrm{H}), 2.14(\mathrm{dtt}, J=$ 11.4, 6.7, $1.2 \mathrm{~Hz}, 1 \mathrm{H}), 1.56(\mathrm{~d}, J=11.4 \mathrm{~Hz}, 1 \mathrm{H}), 1.11(\mathrm{~s}, 9 \mathrm{H}), 0.01(\mathrm{~s}$, $9 \mathrm{H}) .{ }^{13} \mathrm{C}$ NMR $\left(75 \mathrm{MHz}, \mathrm{CDCl}_{3}\right): \delta 147.7,139.2,138.9,135.7,135.7$,
135.6, 133.7, 133.7, 133.2, 129.6, 127.6, 127.6, 124.3, 123.5, 60.4, 48.5, 46.4, 30.8, 26.9, 19.3, 0.4. HRMS (ESI+): $m / z$ calcd for $\mathrm{C}_{29} \mathrm{H}_{38} \mathrm{NaOSi}_{2}$ $[\mathrm{M}+\mathrm{Na}]^{+}, 481.2353$; found, 481.2378.

tert-Butyl(((1 $\left.R^{*}, 6 S^{*}\right)$-8-iodobicyclo[4.2.1]nona-2,4,7-trien-7yl)methoxy)diphenylsilane (27). $N$-Iodosuccinimide ( $2.35 \mathrm{~g}, 10.45$ mmol) was added to a solution of $26(4.00 \mathrm{~g}, 8.72 \mathrm{mmol})$ in anhydrous $\mathrm{CH}_{3} \mathrm{CN}(87 \mathrm{~mL})$ under argon in darkness, and the resulting mixture was stirred at room temperature for $16 \mathrm{~h}$. The reaction was quenched by the addition of a saturated solution of $\mathrm{Na}_{2} \mathrm{~S}_{2} \mathrm{O}_{3}(50 \mathrm{~mL})$ and the product extracted with EtOAc $(3 \times 60 \mathrm{~mL})$. The combined organic layers were dried over $\mathrm{MgSO}_{4}$, filtered, and concentrated under reduced pressure. Purification by flash chromatography (cyclohexane/EtOAc 95/5) afforded the product as a pale yellow oil $(2.52 \mathrm{~g}$, $4.88 \mathrm{mmol}$, yield $56 \%) .{ }^{33}{ }^{1} \mathrm{H}$ NMR $(500 \mathrm{MHz}$, $\left.\mathrm{CDCl}_{3}\right): \delta 7.73-7.66(\mathrm{~m}, 4 \mathrm{H}), 7.48-7.38(\mathrm{~m}, 6 \mathrm{H}), 6.20(\mathrm{ddq}, J=$ $11.2,7.2,1.1 \mathrm{~Hz}, 1 \mathrm{H}), 6.11(\mathrm{ddq}, J=11.2,7.3,1.0 \mathrm{~Hz}, 1 \mathrm{H}), 5.95$ (ddd, $J=11.1,7.5,0.9 \mathrm{~Hz}, 1 \mathrm{H}), 5.86(\mathrm{ddd}, J=11.0,7.4,0.9 \mathrm{~Hz}, 1 \mathrm{H}), 4.35$ (d, $J=13.6,0.8 \mathrm{~Hz}, 1 \mathrm{H}), 4.17(\mathrm{~d}, J=13.5 \mathrm{~Hz}, 1 \mathrm{H}), 3.50(\mathrm{t}, J=7.0 \mathrm{~Hz}$, $1 \mathrm{H}), 3.25(\mathrm{t}, J=7.0 \mathrm{~Hz}, 1 \mathrm{H}), 2.31(\mathrm{dtt}, J=11.3,6.8,1.2 \mathrm{~Hz}, 1 \mathrm{H}), 1.62$ (d, $J=11.4 \mathrm{~Hz}, 1 \mathrm{H}), 1.10$ (s, 9H). ${ }^{13} \mathrm{C} \mathrm{NMR}\left(101 \mathrm{MHz}, \mathrm{CDCl}_{3}\right): \delta$ $145.7,139.1,138.1,135.6,135.6,133.4,133.4,129.7,127.7,125.4$, 124.9, 84.5, 63.0, 52.9, 44.1, 30.8, 26.9, 19.3. HRMS (ESI + ): $m / z$ calcd for $\mathrm{C}_{26} \mathrm{H}_{29} \mathrm{INaOSi}[\mathrm{M}+\mathrm{Na}]^{+}$, 535.0925; found, 535.0919.

$\left(\left(1 R^{*}, 6 S^{*}\right)-8\right.$-(3-(Trimethylsilyl)prop-2-yn-1-yl)bicyclo[4.2.1]nona-2,4,7-trien-7-yl)methanol (29). A dry two-neck roundbottom flask equipped with a condenser was charged with activated magnesium tunings $(583 \mathrm{mg}, 24.0 \mathrm{mmol})$ that were covered with anhydrous THF $(100 \mathrm{~mL})$. Dibromoethane $(0.1 \mathrm{~mL})$ was added, followed by trimethylsilylpropargyl bromide $(2.0 \mathrm{~mL}, 12.12 \mathrm{mmol})$. The reaction mixture was heated at $50{ }^{\circ} \mathrm{C}$ for $1 \mathrm{~h}$ and then cooled to room temperature and transferred via cannula to a second two-neck round-bottom flask containing a solution of $27(1.23 \mathrm{~g}, 2.40 \mathrm{mmol})$ and $\mathrm{Pd}\left(\mathrm{PPh}_{3}\right)_{4}(138.7 \mathrm{mg}, 0.12 \mathrm{mmol})$ in anhydrous THF $(30 \mathrm{~mL})$. The resulting mixture was stirred at $50{ }^{\circ} \mathrm{C}$ for $2 \mathrm{~h}$ and then cooled to room temperature, poured on brine $(100 \mathrm{~mL})$, and extracted with $\mathrm{Et}_{2} \mathrm{O}(2 \times 100 \mathrm{~mL})$. The combined organic layers were dried over $\mathrm{MgSO}_{4}$, filtered, and concentrated under reduced pressure. Purification by flash chromatography (cyclohexane/EtOAc 95/5) afforded 28 as a yellow oil that was directly taken to the next step due to its low stability. ${ }^{1} \mathrm{H}$ NMR $\left(400 \mathrm{MHz}, \mathrm{CDCl}_{3}\right): \delta 7.72-7.67(\mathrm{~m}, 4 \mathrm{H}), 7.47-$ $7.36(\mathrm{~m}, 6 \mathrm{H}), 6.27-6.18(\mathrm{~m}, 1 \mathrm{H}), 6.17-6.09(\mathrm{~m}, 1 \mathrm{H}), 5.83-5.71(\mathrm{~m}$, $2 \mathrm{H}), 4.33(\mathrm{~d}, J=12.8 \mathrm{~Hz}, 1 \mathrm{H}), 4.20(\mathrm{~d}, J=13.0 \mathrm{~Hz}, 1 \mathrm{H}), 3.42(\mathrm{t}, J=$ $7.0 \mathrm{~Hz}, 1 \mathrm{H}), 3.36(\mathrm{t}, J=7.0 \mathrm{~Hz}, 1 \mathrm{H}), 2.95(\mathrm{~d}, J=19.4 \mathrm{~Hz}, 1 \mathrm{H}), 2.85$ $(\mathrm{d}, J=18.6 \mathrm{~Hz}, 1 \mathrm{H}), 2.26(\mathrm{dtt}, J=11.3,6.7,1.2 \mathrm{~Hz}, 1 \mathrm{H}), 1.61(\mathrm{~d}, J=$ $11.3 \mathrm{~Hz}, 1 \mathrm{H}), 1.08(\mathrm{~s}, 9 \mathrm{H}), 0.16(\mathrm{~s}, 9 \mathrm{H}) .{ }^{13} \mathrm{C}$ NMR $(101 \mathrm{MHz}$, $\left.\mathrm{CDCl}_{3}\right): \delta 140.2,139.7,135.6,135.6,135.0,133.7,133.6,129.9,129.6$, $129.6,127.7,127.7,124.4,124.1,104.8,85.0,58.5,46.6,45.0,30.3$, $26.8,19.2,17.2,0.1$. To a solution of $28(646.0 \mathrm{mg}, 1.30 \mathrm{mmol})$ in THF $(12 \mathrm{~mL})$ in a Teflon flask was added HF.py (70\% weight, 0.31 $\mathrm{mL}, 12.1 \mathrm{mmol})$, and the resulting mixture was stirred at room temperature for $16 \mathrm{~h}$. The reaction was quenched by the slow addition of a saturated solution of $\mathrm{NaHCO}_{3}(10 \mathrm{~mL})$, and the product was extracted with $\mathrm{Et}_{2} \mathrm{O}(2 \times 10 \mathrm{~mL})$. The combined organic layers were dried over $\mathrm{MgSO}_{4}$, filtered, and concentrated under reduced pressure. Purification by flash chromatography (cyclohexane/EtOAc 8/2) afforded the product as a yellow oil $(528.1 \mathrm{mg}, 1.06 \mathrm{mmol}$, yield over two steps 44\%). ${ }^{1} \mathrm{H}$ NMR (400 $\left.\mathrm{MHz}, \mathrm{CDCl}_{3}\right): \delta 6.31-6.19(\mathrm{~m}$, $2 \mathrm{H}), 5.87-5.78(\mathrm{~m}, 2 \mathrm{H}), 4.29(\mathrm{~d}, J=12.7 \mathrm{~Hz}, 1 \mathrm{H}), 4.19(\mathrm{~d}, J=12.7$ $\mathrm{Hz}, 1 \mathrm{H}), 3.37(\mathrm{~d}, J=7.2 \mathrm{~Hz}, 1 \mathrm{H}), 3.34(\mathrm{~d}, J=7.2 \mathrm{~Hz}, 1 \mathrm{H}), 3.17(\mathrm{~d}, J$ $=19.3 \mathrm{~Hz}, 1 \mathrm{H}), 3.11(\mathrm{~d}, J=19.1 \mathrm{~Hz}, 1 \mathrm{H}), 2.27(\mathrm{dtt}, J=11.4,6.7,1.2$ $\mathrm{Hz}, 1 \mathrm{H}), 1.61(\mathrm{~d}, J=11.4 \mathrm{~Hz}, 1 \mathrm{H}), 0.18(\mathrm{~s}, 9 \mathrm{H}) .{ }^{13} \mathrm{C}$ NMR $(101$ $\left.\mathrm{MHz}, \mathrm{CDCl}_{3}\right): \delta 139.8,139.4,135.1,131.5,124.7,124.5,104.4,85.4$, 57.3, 46.7, 45.4, 30.1, 17.2, 0.0. HRMS (ESI+): $\mathrm{m} / \mathrm{z}$ calcd for $\mathrm{C}_{16} \mathrm{H}_{22} \mathrm{NaOSi}[\mathrm{M}+\mathrm{Na}]^{+}$, 281.1332; found, 281.1322.

$\left(\left(1 R^{*}, 6 S^{*}, 7 R^{*}, 9 S^{*}\right)-9\right.$-(3-(Trimethylsilyl)prop-2-yn-1-yl)tricyclo[4.3.1.0 ${ }^{7,9}$ ]deca-2,4-dien-7-yl)methanol (30). To a solution of $29(430 \mathrm{mg}, 1.66 \mathrm{mmol})$ in anhydrous $\mathrm{CH}_{2} \mathrm{Cl}_{2}(83 \mathrm{~mL})$ were sequentially added $\mathrm{CH}_{2} \mathrm{I}_{2}(0.15 \mathrm{~mL}, 1.99 \mathrm{mmol})$ and $\mathrm{ZnEt}_{2}(1.0 \mathrm{M}$ in hexanes, $4.15 \mathrm{~mL}, 4.15 \mathrm{mmol}$ ), and the resulting solution was stirred at room temperature for $3 \mathrm{~h}$. The reaction mixture was quenched by the 
slow addition of a saturated aqueous $\mathrm{Na} / \mathrm{K}$-tartrate solution $(100 \mathrm{~mL})$, and after the mixture was stirred for $30 \mathrm{~min}$, the organic layer was separated, the aqueous layer was extracted with $\mathrm{CH}_{2} \mathrm{Cl}_{2}(100 \mathrm{~mL})$, and the combined organic phases were dried over $\mathrm{MgSO}_{4}$ and concentrated under reduced pressure. Purification by flash chromatography (cyclohexane/EtOAc $8 / 2$ ) afforded the product as a pale yellow oil (378.0 mg, $1.39 \mathrm{mmol}$, yield $84 \%) .{ }^{1} \mathrm{H}$ NMR $\left(400 \mathrm{MHz}, \mathrm{CDCl}_{3}\right): \delta$ 6.11-6.00 (m, $2 \mathrm{H}), 5.84-5.70(\mathrm{~m}, 2 \mathrm{H}), 3.92(\mathrm{~d}, J=11.6 \mathrm{~Hz}, 1 \mathrm{H})$, $3.57(\mathrm{~d}, J=11.5 \mathrm{~Hz}, 1 \mathrm{H}), 2.89$ (dd, $J=17.3,0.8 \mathrm{~Hz}, 1 \mathrm{H}), 2.85-2.75$ (m, 2H), 2.07 (d, $J=17.3 \mathrm{~Hz}, 1 \mathrm{H}), 1.99$ (ddd, $J=12.8,7.0,5.7 \mathrm{~Hz}$, $1 \mathrm{H}), 1.74(\mathrm{~d}, J=13.1 \mathrm{~Hz}, 1 \mathrm{H}), 1.50(\mathrm{~s}, 1 \mathrm{H}), 0.74(\mathrm{~d}, J=5.7 \mathrm{~Hz}, 1 \mathrm{H})$, $0.18(\mathrm{~s}, 10 \mathrm{H}) .{ }^{13} \mathrm{C}$ NMR $\left(101 \mathrm{MHz}, \mathrm{CDCl}_{3}\right): \delta 136.9,136.5,125.7$, 125.3, 105.4, 85.8, 64.0, 43.1, 42.3, 41.4, 38.9, 26.2, 21.0, 14.3, 0.1 . HRMS (ESI+): $m / z$ calcd for $\mathrm{C}_{17} \mathrm{H}_{24} \mathrm{NaOSi}[\mathrm{M}+\mathrm{Na}]^{+}, 295.1489$; found, 295.1482 .

tert-Butyldimethyl $\left(\left(\left(1 R^{*}, 6 S^{*}, 7 R^{*}, 9 R^{*}\right)\right.\right.$-9-(prop-2-yn-1-yl)tricyclo[4.3.1.0 $\left.0^{7,9}\right]$ deca-2,4-dien-7-yl)methoxy)silane (4a). TBAF (1.0 M solution in THF, $1.53 \mathrm{~mL}, 1.53 \mathrm{mmol}$ ) was added to a solution of $30(378.1 \mathrm{mg}, 1.39 \mathrm{mmol})$ in THF $(14 \mathrm{~mL})$ at $0{ }^{\circ} \mathrm{C}$, and the resulting solution was warmed to room temperature and stirred for $15 \mathrm{~min}$. Then the mixture was poured on brine $(30 \mathrm{~mL})$ and the product was extracted with $\mathrm{Et}_{2} \mathrm{O}(2 \times 20 \mathrm{~mL})$. The combined organic layers were dried over $\mathrm{MgSO}_{4}$, filtered, and concentrated under reduced pressure. Purification by flash chromatography (cyclohexane/ EtOAc 8/2) afforded $\left(\left(1 R^{*}, 6 S^{*}, 7 R^{*}, 9 R^{*}\right)\right.$-9-(prop-2-yn-1-yl)tricyclo[4.3.1.0 $\left.0^{7,9}\right]$ deca-2,4-dien-7-yl)methanol $\left(\mathbf{3 0}^{\prime}\right)$ as a colorless oil $(222.7$ mg, $1.11 \mathrm{mmol}$, yield $80 \%)$. ${ }^{1} \mathrm{H}$ NMR $\left(400 \mathrm{MHz}, \mathrm{CDCl}_{3}\right): \delta 6.12-$ $6.03(\mathrm{~m}, 2 \mathrm{H}), 5.84-5.76(\mathrm{~m}, 2 \mathrm{H}), 3.91(\mathrm{~d}, J=11.6 \mathrm{~Hz}, 1 \mathrm{H}), 3.60(\mathrm{~d}, J$ $=11.6 \mathrm{~Hz}, 1 \mathrm{H}), 2.91(\mathrm{ddd}, J=17.1,2.7,0.9 \mathrm{~Hz}, 1 \mathrm{H}), 2.84(\mathrm{td}, J=6.8$, $4.0 \mathrm{~Hz}, 2 \mathrm{H}), 2.04(\mathrm{t}, J=2.7 \mathrm{~Hz}, 1 \mathrm{H}), 2.03-1.95(\mathrm{~m}, 2 \mathrm{H}), 1.75(\mathrm{~d}, J=$ $13.1 \mathrm{~Hz}, 1 \mathrm{H}), 1.53(\mathrm{~s}, 1 \mathrm{H}), 0.76(\mathrm{~d}, J=5.7 \mathrm{~Hz}, 1 \mathrm{H}), 0.17(\mathrm{~d}, J=5.7$ $\mathrm{Hz}, 1 \mathrm{H}) .{ }^{13} \mathrm{C}$ NMR $\left(101 \mathrm{MHz}, \mathrm{CDCl}_{3}\right): \delta 136.9,136.4,125.8,125.3$, 82.8, 69.1, 64.1, 42.8, 42.3, 41.6, 38.8, 26.1, 19.7, 14.3. HRMS (APCI $+): m / z$ calcd for $\mathrm{C}_{14} \mathrm{H}_{17} \mathrm{O}[\mathrm{M}+\mathrm{H}]^{+}, 201.1274$; found, 201.1266. TBSCl $(165.6 \mathrm{mg}, 1.10 \mathrm{mmol})$ and imidazole $(136.2 \mathrm{mg}, 2.00 \mathrm{mmol})$ were added to a solution of $30^{\prime}(200.3 \mathrm{mg}, 1.00 \mathrm{mmol})$ in $\mathrm{CH}_{2} \mathrm{Cl}_{2}(10$ $\mathrm{mL}$ ), and the mixture was stirred at room temperature for $1 \mathrm{~h}$. Then it was washed with $\mathrm{H}_{2} \mathrm{O}(10 \mathrm{~mL})$ and brine $(10 \mathrm{~mL})$ and the organic layer was dried over $\mathrm{MgSO}_{4}$, filtered, and concentrated under reduced pressure. Purification by column chromatography (cyclohexane) afforded the product as a colorless oil $(314.4 \mathrm{mg}, 0.99 \mathrm{mmol}$, yield 99\%). ${ }^{1} \mathrm{H}$ NMR $\left(400 \mathrm{MHz}, \mathrm{CDCl}_{3}\right): \delta 6.06-5.92(\mathrm{~m}, 2 \mathrm{H}), 5.79-5.69$ $(\mathrm{m}, 2 \mathrm{H}), 4.08(\mathrm{dd}, J=10.4,1.1 \mathrm{~Hz}, 1 \mathrm{H}), 3.37(\mathrm{~d}, J=10.4 \mathrm{~Hz}, 1 \mathrm{H})$, $2.85-2.73(\mathrm{~m}, 3 \mathrm{H}), 2.04-1.94(\mathrm{~m}, 3 \mathrm{H}), 1.72(\mathrm{~d}, J=13.0 \mathrm{~Hz}, 1 \mathrm{H})$, $0.91(\mathrm{~s}, 9 \mathrm{H}), 0.71(\mathrm{~d}, J=5.6 \mathrm{~Hz}, 1 \mathrm{H}), 0.22(\mathrm{~d}, J=5.7 \mathrm{~Hz}, 1 \mathrm{H}), 0.06$ $(\mathrm{s}, 3 \mathrm{H}), 0.04(\mathrm{~s}, 3 \mathrm{H}) .{ }^{13} \mathrm{C}$ NMR $\left(101 \mathrm{MHz}, \mathrm{CDCl}_{3}\right): \delta 136.8,136.4$, 125.3, 124.9, 83.2, 68.8, 63.0, 43.1, 42.4, 41.5, 38.3, 26.0, 25.9, 19.1, $18.3,13.6,-5.2,-5.4$. HRMS (ESI + ): $m / z$ calcd for $\mathrm{C}_{20} \mathrm{H}_{30} \mathrm{NaOSi}[\mathrm{M}$ $+\mathrm{Na}]^{+}, 337.1958$; found, 337.1945.

$1-\left(\left(1 R^{*}, 6 S^{*}, 7 R^{*}, 9 S^{*}\right)-9-(((\right.$ tert-Butyldimethylsilyl)oxy)methyl)tricyclo[4.3.1.0 $0^{7,9}$ ]deca-2,4-dien-7-yl)propan-2-one (31). Gold(I) complex $(0.002 \mathrm{mmol})$ was added to a solution of $4 \mathrm{a}$ $(0.1 \mathrm{mmol})$ in $\mathrm{MeOH}(1 \mathrm{~mL})$, and the resulting mixture was stirred at room temperature for $2 \mathrm{~h}$ before the addition of 1 drop of $\mathrm{Et}_{3} \mathrm{~N}$. Then the volatiles were removed under reduced pressure, and purification by column chromatography afforded the product in $22-47 \%$ yield. ${ }^{1} \mathrm{H}$ NMR (400 MHz, CDCl $): \delta 5.98-5.83(\mathrm{~m}, 2 \mathrm{H}), 5.77-5.69(\mathrm{~m}, 2 \mathrm{H})$, $4.09(\mathrm{~d}, J=10.6 \mathrm{~Hz}, 1 \mathrm{H}), 3.37(\mathrm{~d}, J=10.4 \mathrm{~Hz}, 1 \mathrm{H}), 3.01(\mathrm{~d}, J=16.1$ $\mathrm{Hz}, 1 \mathrm{H}), 2.76(\mathrm{t}, J=7.2 \mathrm{~Hz}, 1 \mathrm{H}), 2.73(\mathrm{t}, J=6.7 \mathrm{~Hz}, 1 \mathrm{H}), 2.18(\mathrm{~s}$, $3 \mathrm{H}), 2.14(\mathrm{~d}, J=16.0 \mathrm{~Hz}, 1 \mathrm{H}), 2.04-1.95(\mathrm{~m}, 1 \mathrm{H}), 1.71(\mathrm{~d}, J=13.0$ $\mathrm{Hz}, 1 \mathrm{H}), 0.91(\mathrm{~s}, 9 \mathrm{H}), 0.79(\mathrm{~d}, J=5.7 \mathrm{~Hz}, 1 \mathrm{H}), 0.21(\mathrm{~d}, J=5.7 \mathrm{~Hz}$, $1 \mathrm{H}), 0.06(\mathrm{~s}, 3 \mathrm{H}), 0.06(\mathrm{~s}, 3 \mathrm{H}) .{ }^{13} \mathrm{C}$ NMR $\left(101 \mathrm{MHz}, \mathrm{CDCl}_{3}\right): \delta$ 208.9, 136.6, 136.5, 125.2, 125.1, 63.1, 43.7, 42.9, 41.8, 41.0, 35.6, 30.2, $26.4,25.9,18.3,13.9,-5.2,-5.4$. HRMS (ESI $): \mathrm{m} / \mathrm{z}$ calcd for $\mathrm{C}_{20} \mathrm{H}_{32} \mathrm{NaO}_{2} \mathrm{Si}[\mathrm{M}+\mathrm{Na}]^{+}$, 355.2064; found, 355.2056.

Synthesis of $\mathbf{4 b}, \mathbf{c}$. Ethynylmagnesium bromide $(0.5 \mathrm{M}$ in THF, $8.07 \mathrm{~mL}, 4.04 \mathrm{mmol})$ was added to a solution of $9(3.67 \mathrm{mmol})$ in anhydrous THF $(37 \mathrm{~mL})$ at $0{ }^{\circ} \mathrm{C}$. After it was stirred at room temperature for $30 \mathrm{~min}$, the reaction mixture was diluted with $\mathrm{Et}_{2} \mathrm{O}$ $(15 \mathrm{~mL})$ and quenched by the addition of saturated $\mathrm{NH}_{4} \mathrm{Cl}$ aqueous solution $(50 \mathrm{~mL})$. The aqueous layer was extracted with $\mathrm{Et}_{2} \mathrm{O}(2 \times 40$ $\mathrm{mL}$ ), the combined organic phases were dried over $\mathrm{MgSO}_{4}$, filtered, and concentrated under reduced pressure, and the resulting crude was purified by column chromatography (cyclohexane/EtOAc $7 / 3$ ).

1-((1R*,6S*,7R*,9S*)-9-(Methoxymethyl)tricyclo[4.3.1.0 $\left.0^{7,9}\right]$ deca2,4-dien-7-yl)prop-2-yn-1-ol (4b). Pale yellow solid (769.2 mg, 3.34 mmol, yield 91\%). Mp: 82-84 ${ }^{\circ} \mathrm{C} .{ }^{1} \mathrm{H}$ NMR $\left(400 \mathrm{MHz}, \mathrm{CDCl}_{3}\right): \delta$ 6.10-5.99 (m, 2H), 5.84-5.72 (m, 2H), $4.94(\mathrm{~s}, 1 \mathrm{H}), 3.87(\mathrm{dd}, J=$ 10.1, $0.7 \mathrm{~Hz}, 1 \mathrm{H}), 3.68(\mathrm{~d}, J=10.1 \mathrm{~Hz}, 1 \mathrm{H}), 3.37(\mathrm{~s}, 3 \mathrm{H}), 2.97(\mathrm{~s}$, $1 \mathrm{H}), 2.82(\mathrm{dd}, J=7.1,6.2 \mathrm{~Hz}, 1 \mathrm{H}), 2.76(\mathrm{dd}, J=7.1,6.2 \mathrm{~Hz}, 1 \mathrm{H}), 2.54$ $(\mathrm{d}, J=2.2 \mathrm{~Hz}, 1 \mathrm{H}), 2.12-2.03(\mathrm{~m}, 1 \mathrm{H}), 1.76(\mathrm{~d}, J=13.0 \mathrm{~Hz}, 1 \mathrm{H})$, $0.76(\mathrm{~d}, J=5.9 \mathrm{~Hz}, 1 \mathrm{H}), 0.74(\mathrm{~d}, J=6.1 \mathrm{~Hz}, 1 \mathrm{H}) .{ }^{13} \mathrm{C}$ NMR $(101$ $\left.\mathrm{MHz} \mathrm{CDCl}_{3}\right): \delta 137.2,136.0,126.2,125.0,84.0,73.5,72.0,61.1,58.7$, 43.8, 43.3, 43.0, 40.2, 26.3, 10.6. HRMS (APCI+): $m / z$ calcd for $\mathrm{C}_{15} \mathrm{H}_{18} \mathrm{NaO}_{2}[\mathrm{M}+\mathrm{Na}]^{+}$, 253.1199; found, 253.1211.

1-((1R*,6S*,7R*,9S*)-9-((Benzyloxy)methyl)tricyclo[4.3.1.0 $\left.{ }^{7,9}\right]$ deca-2,4-dien-7-yl)prop-2-yn-1-ol (4c). Colorless oil (923.0 mg, 3.01 mmol, yield $82 \%) .{ }^{1} \mathrm{H}$ NMR (500 $\left.\mathrm{MHz}, \mathrm{CDCl}_{3}\right): \delta 7.39-7.33(\mathrm{~m}$, $4 \mathrm{H}), 7.33-7.29(\mathrm{~m}, 1 \mathrm{H}), 6.06(\mathrm{dd}, J=10.7,7.5 \mathrm{~Hz}, 1 \mathrm{H}), 6.00(\mathrm{dd}, J=$ 9.7, $7.5 \mathrm{~Hz}, 1 \mathrm{H}), 5.80$ (ddd, $J=11.3,7.4,0.9 \mathrm{~Hz}, 1 \mathrm{H}), 5.74(\mathrm{ddd}, J=$ $11.2,7.4,0.9 \mathrm{~Hz}, 1 \mathrm{H}), 4.95(\mathrm{dd}, J=4.0,2.2 \mathrm{~Hz}, 1 \mathrm{H}), 4.60$ (d, $J=11.9$ $\mathrm{Hz}, 1 \mathrm{H}), 4.51(\mathrm{~d}, J=11.9 \mathrm{~Hz}, 1 \mathrm{H}), 3.97(\mathrm{~d}, J=10.2 \mathrm{~Hz}, 1 \mathrm{H}), 3.80(\mathrm{~d}$, $J=10.2 \mathrm{~Hz}, 1 \mathrm{H}), 2.89(\mathrm{~d}, J=4.1 \mathrm{~Hz}, 1 \mathrm{H}), 2.85-2.79(\mathrm{~m}, 2 \mathrm{H}), 2.51$ $(\mathrm{d}, J=2.2 \mathrm{~Hz}, 1 \mathrm{H}), 2.08(\mathrm{dtt}, J=12.8,6.3,1.3 \mathrm{~Hz}, 1 \mathrm{H}), 1.77(\mathrm{~d}, J=$ $13.1 \mathrm{~Hz}, 1 \mathrm{H}), 0.79-0.75(\mathrm{~m}, 2 \mathrm{H}) .{ }^{13} \mathrm{C}$ NMR $\left(126 \mathrm{MHz}, \mathrm{CDCl}_{3}\right): \delta$ 138.1, 137.3, 136.0, 128.4, 127.7, 127.6, 126.3, 125.0, 83.9, 73.6, 73.0, 69.7, 61.2, 43.8, 43.3, 43.0, 40.3, 26.4, 11.0. HRMS (ESI+): $\mathrm{m} / z$ calcd for $\mathrm{C}_{21} \mathrm{H}_{22} \mathrm{NaO}_{2}[\mathrm{M}+\mathrm{Na}]^{+}$, 329.1512; found, 329.1518 .

Gold-Catalyzed Cyclization of 1,6-Enynes 4b,c. Gold(I) complex B $(3.1 \mathrm{mg}, 0.002 \mathrm{mmol})$ was added to a solution of $4 \mathbf{b}, \mathbf{c}$ $(0.1 \mathrm{mmol})$ in $\mathrm{ROH}(1 \mathrm{~mL})$, and the resulting suspension was stirred at room temperature for $2 \mathrm{~h}$ before the addition of 1 drop of $\mathrm{Et}_{3} \mathrm{~N}$. Then the volatiles were removed under reduced pressure and the resulting crude was purified by column chromatography to afford tetracycles 32

$(1 a R *, 2 S * 3 a S * 4 S * 6 R * 6 a S *, 7 R *)-6,7-D i m e t h o x y-1 a-$ (methoxymethyl)-5-methylene-1,1a,2,3,3a,4,5,6-octahydro-2,4-prop[1]enocyclopropa[c]pentalene (32a). Purification: pentane $/ \mathrm{CH}_{2} \mathrm{Cl}_{2}$ 9/ 1. White solid (25.1 mg, yield 91\%). 32a:32a' > 20:1. Mp: $95-97^{\circ} \mathrm{C}$. ${ }^{1} \mathrm{H}$ NMR $\left(500 \mathrm{MHz}, \mathrm{CDCl}_{3}\right): \delta 6.12(\mathrm{ddd}, J=11.6,6.8,1.5 \mathrm{~Hz}, 1 \mathrm{H})$, $5.76(\mathrm{ddd}, J=11.6,7.3,0.6 \mathrm{~Hz}, 1 \mathrm{H}), 5.17(\mathrm{~d}, J=2.9 \mathrm{~Hz}, 1 \mathrm{H}), 5.04(\mathrm{~d}$, $J=2.4 \mathrm{~Hz}, 1 \mathrm{H}), 3.98(\mathrm{dd}, J=7.3,3.7 \mathrm{~Hz}, 1 \mathrm{H}), 3.70(\mathrm{dd}, J=9.8,1.6$ $\mathrm{Hz}, 1 \mathrm{H}), 3.47(\mathrm{~s}, 1 \mathrm{H}), 3.41(\mathrm{~s}, 3 \mathrm{H}), 3.36(\mathrm{~s}, 3 \mathrm{H}), 3.36-3.29(\mathrm{~m}, 1 \mathrm{H})$, $3.33(\mathrm{~s}, 3 \mathrm{H}), 2.90(\mathrm{dd}, J=9.7,6.6 \mathrm{~Hz}, 1 \mathrm{H}), 2.84-2.80(\mathrm{~m}, 2 \mathrm{H}), 2.50$ $(\mathrm{d}, J=13.7 \mathrm{~Hz}, 1 \mathrm{H}), 1.42(\mathrm{dtd}, J=13.6,6.7,1.6 \mathrm{~Hz}, 1 \mathrm{H}), 1.12(\mathrm{dd}, J=$ $5.8,1.6 \mathrm{~Hz}, 1 \mathrm{H}), 1.08(\mathrm{~d}, J=5.8 \mathrm{~Hz}, 1 \mathrm{H}) .{ }^{13} \mathrm{C}$ NMR $(101 \mathrm{MHz}$, $\left.\mathrm{CDCl}_{3}\right): \delta 151.2,140.2,127.6,110.9,85.7,80.8,74.1,58.7,56.6,55.6$, 45.9, 45.6, 42.8, 40.9, 36.1, 30.3, 16.0. HRMS (ESI+): $\mathrm{m} / z$ calcd for $\mathrm{C}_{17} \mathrm{H}_{24} \mathrm{NaO}_{3}[\mathrm{M}+\mathrm{Na}]^{+}, 299.1618$; found, 299.1626. Note: this reaction could be scaled up to obtain $400 \mathrm{mg}$ of 32a without any decrease in yield or selectivity. X-ray-quality single crystals were obtained by slow evaporation of a solution of 32a in $\mathrm{CH}_{2} \mathrm{Cl}_{2}$ at $5{ }^{\circ} \mathrm{C}$.

$(1 a R *, 2 S *, 3 a S *, 4 S *, 6 R *, 6 a S *, 7 R *)-1 a-(($ Benzyloxy)methyl)-6,7dimethoxy-5-methylene-1,1a,2,3,3a,4,5,6-octahydro-2,4-prop[1]enocyclopropa[c]pentalene (32b). Purification: cyclohexane/EtOAc $1 / 0$ to $95 / 5$. Colorless oil $(29.6 \mathrm{mg}$, yield $84 \%) . \mathbf{3 2 b}: 32 \mathbf{b}^{\prime}=5: 1$. Data for the major isomer are as follows. ${ }^{1} \mathrm{H}$ NMR $\left(500 \mathrm{MHz}, \mathrm{CDCl}_{3}\right): \delta$ $7.40-7.34(\mathrm{~m}, 4 \mathrm{H}), 7.33-7.29(\mathrm{~m}, 1 \mathrm{H}), 6.09$ (ddd, $J=11.6,6.7,1.5$ $\mathrm{Hz}, 1 \mathrm{H}), 5.72(\mathrm{ddd}, J=11.6,7.3,0.6 \mathrm{~Hz}, 1 \mathrm{H}), 5.15(\mathrm{~d}, J=2.9 \mathrm{~Hz}$, $1 \mathrm{H}), 5.03(\mathrm{~d}, J=2.4 \mathrm{~Hz}, 1 \mathrm{H}), 4.57(\mathrm{~d}, J=12.1 \mathrm{~Hz}, 1 \mathrm{H}), 4.52(\mathrm{~d}, J=$ $12.1 \mathrm{~Hz}, 1 \mathrm{H}), 3.97(\mathrm{dd}, J=7.3,3.7 \mathrm{~Hz}, 1 \mathrm{H}), 3.78(\mathrm{dd}, J=9.8,1.5 \mathrm{~Hz}$, $1 \mathrm{H}), 3.45(\mathrm{~d}, J=1.2 \mathrm{~Hz}, 1 \mathrm{H}), 3.41(\mathrm{~s}, 3 \mathrm{H}), 3.32(\mathrm{~s}, 1 \mathrm{H}), 3.32(\mathrm{~s}, 3 \mathrm{H})$, $2.96(\mathrm{~d}, J=9.8 \mathrm{~Hz}, 1 \mathrm{H}), 2.91(\mathrm{dd}, J=10.0,6.3 \mathrm{~Hz}, 1 \mathrm{H}), 2.87(\mathrm{t}, J=$ $6.9 \mathrm{~Hz}, 1 \mathrm{H}), 2.51(\mathrm{~d}, J=13.7 \mathrm{~Hz}, 1 \mathrm{H}), 1.47-1.40(\mathrm{~m}, 1 \mathrm{H}), 1.14(\mathrm{dd}, J$ $=5.8,1.5 \mathrm{~Hz}, 1 \mathrm{H}), 1.10(\mathrm{~d}, J=5.8 \mathrm{~Hz}, 1 \mathrm{H}) .{ }^{13} \mathrm{C}$ NMR $(126 \mathrm{MHz}$, $\left.\mathrm{CDCl}_{3}\right): \delta 151.2,140.2,138.5,128.4,128.4,127.6,127.6,110.9,85.7$, 80.8, 72.7, 71.5, 56.6, 55.6, 46.0, 45.7, 42.9, 41.0, 36.3, 30.3, 16.1 . HRMS (ESI +$): m / z$ calcd for $\mathrm{C}_{23} \mathrm{H}_{28} \mathrm{NaO}_{3}[\mathrm{M}+\mathrm{Na}]^{+}, 375.1931$; found, 375.1925 . 
$(1 a R *, 2 S *, 3 a S *, 4 S *, 6 R *, 6 a S *, 7 R *)-6,7-B i s($ allyloxy) $-1 a-$ ((benzyloxy)methyl)-5-methylene-1,1a,2,3,3a,4,5,6-octahydro-2,4prop[1]enocyclopropa[c]pentalene (32c). Purification: cyclohexane/ EtOAc $1 / 0$ to $95 / 5$. White solid (30.0 mg, yield $84 \%) .32 \mathrm{c}: 32 \mathrm{c}^{\prime}>$ 20:1. Mp: $138-140{ }^{\circ} \mathrm{C} .{ }^{1} \mathrm{H}$ NMR $\left(500 \mathrm{MHz}, \mathrm{CDCl}_{3}\right): \delta 7.39-7.34$ (m, 4H), 7.33-7.29 (m, 1H), 6.07 (ddd, $J=11.6,6.8,1.5 \mathrm{~Hz}, 1 \mathrm{H})$, $6.01-5.88(\mathrm{~m}, 2 \mathrm{H}), 5.69(\mathrm{ddd}, J=11.6,7.3,0.6 \mathrm{~Hz}, 1 \mathrm{H}), 5.31(\mathrm{dq}, J=$ $17.2,1.7 \mathrm{~Hz}, 1 \mathrm{H}), 5.26(\mathrm{dq}, J=17.2,1.7 \mathrm{~Hz}, 1 \mathrm{H}), 5.20(\mathrm{dq}, J=10.4$, $1.8 \mathrm{~Hz}, 1 \mathrm{H}), 5.17(\mathrm{dq}, J=10.2,1.9 \mathrm{~Hz}, 1 \mathrm{H}), 5.13(\mathrm{~d}, J=2.9 \mathrm{~Hz}, 1 \mathrm{H})$, $4.99(\mathrm{~d}, J=2.4 \mathrm{~Hz}, 1 \mathrm{H}), 4.56(\mathrm{~d}, J=12.1 \mathrm{~Hz}, 1 \mathrm{H}), 4.51(\mathrm{~d}, J=12.1$ $\mathrm{Hz}, 1 \mathrm{H}), 4.13(\mathrm{dd}, J=7.4,3.8 \mathrm{~Hz}, 1 \mathrm{H}), 4.09(\mathrm{dt}, J=5.5,1.5 \mathrm{~Hz}, 1 \mathrm{H})$, $4.08(\mathrm{dt}, J=5.7,1.5 \mathrm{~Hz}, 1 \mathrm{H}), 4.05(\mathrm{dq}, J=5.7,1.6 \mathrm{~Hz}, 1 \mathrm{H}), 3.97(\mathrm{ddt}$, $J=12.7,6.0,1.4 \mathrm{~Hz}, 1 \mathrm{H}), 3.77(\mathrm{dd}, J=9.8,1.5 \mathrm{~Hz}, 1 \mathrm{H}), 3.65(\mathrm{~s}, 1 \mathrm{H})$, $3.34(\mathrm{dq}, J=9.5,3.0 \mathrm{~Hz}, 1 \mathrm{H}), 2.96(\mathrm{~d}, J=6.3 \mathrm{~Hz}, 1 \mathrm{H}), 2.94(\mathrm{~d}, J=6.4$ $\mathrm{Hz}, 1 \mathrm{H}), 2.87(\mathrm{t}, J=6.7 \mathrm{~Hz}, 1 \mathrm{H}), 2.57(\mathrm{~d}, J=13.7 \mathrm{~Hz}, 1 \mathrm{H}), 1.48-1.42$ $(\mathrm{m}, 1 \mathrm{H}), 1.16(\mathrm{dd}, J=5.8,1.5 \mathrm{~Hz}, 1 \mathrm{H}), 1.09(\mathrm{~d}, J=5.8 \mathrm{~Hz}, 1 \mathrm{H}) .{ }^{13} \mathrm{C}$ NMR $\left(126 \mathrm{MHz}, \mathrm{CDCl}_{3}\right): \delta 151.8,140.1,138.5,135.4,135.3,128.4$, 127.8, 127.6, 127.6, 116.9, 116.8, 110.8, 83.2, 78.2, 72.7, 71.5, 69.7, 68.5, 46.3, 45.7, 42.9, 41.1, 36.3, 30.3, 16.3. HRMS (ESI+): $m / z$ calcd for $\mathrm{C}_{27} \mathrm{H}_{32} \mathrm{NaO}_{3}[\mathrm{M}+\mathrm{Na}]^{+}$, 427.2244; found, 427.2227.

$\left(\left(1 R^{*}, 6 S^{*}, 7 S^{*}, 9 R^{*}\right)-9-\left(\right.\right.$ Methoxymethyl)tricyclo[4.3.1.0 $\left.0^{7,9}\right]-$ decan-7-yl)methanol (33). A round-bottom flask containing a solution of $8 \mathrm{a}(200 \mathrm{mg}, 0.97 \mathrm{mmol})$ and $\mathrm{Pd}(\mathrm{OH})_{2} / \mathrm{C}(20 \mathrm{wt} \%, 28.1$ $\mathrm{mg}, 0.048 \mathrm{mmol})$ in anhydrous $\mathrm{MeOH}(10 \mathrm{~mL})$ was evacuated and back-filled with $\mathrm{H}_{2}$ (repeated three times). The resulting mixture was stirred at room temperature for $4 \mathrm{~h}$, and then the volatiles were removed under reduced pressure and the crude was purified by column chromatography (cyclohexane/EtOAc 1/1) to afford the product as a colorless oil $\left(204.0 \mathrm{mg}, 0.97 \mathrm{mmol}\right.$, yield quantitative). ${ }^{1} \mathrm{H}$ NMR $\left(400 \mathrm{MHz}, \mathrm{CDCl}_{3}\right): \delta 4.02(\mathrm{dd}, J=11.6,1.1 \mathrm{~Hz}, 1 \mathrm{H}), 3.79(\mathrm{dd}$, $J=10.2,1.0 \mathrm{~Hz}, 1 \mathrm{H}), 3.48(\mathrm{~d}, J=11.6 \mathrm{~Hz}, 1 \mathrm{H}), 3.37(\mathrm{~s}, 3 \mathrm{H}), 3.27$ (d, $J=10.1 \mathrm{~Hz}, 1 \mathrm{H}), 2.43-1.36(\mathrm{~m}, 2 \mathrm{H}), 2.01-1.93(\mathrm{~m}, 1 \mathrm{H}), 1.91-1.82$ (m, 1H), 1.70-1.34 (m, 9H), $0.88(\mathrm{dt}, J=4.7,1.1 \mathrm{~Hz}, 1 \mathrm{H}), 0.52(\mathrm{~d}, J$ $=4.6 \mathrm{~Hz}, 1 \mathrm{H}) .{ }^{13} \mathrm{C}$ NMR $\left(126 \mathrm{MHz}, \mathrm{CDCl}_{3}\right): \delta 73.6,63.4,58.8,40.4$, 39.8, 36.3, 34.7, 29.1, 29.1, 28.9, 25.1, 24.8, 18.4. HRMS (ESI+): $m / z$ calcd for $\mathrm{C}_{13} \mathrm{H}_{22} \mathrm{NaO}_{2}[\mathrm{M}+\mathrm{Na}]^{+}$, 233.1512; found, 233.1511.

$\left(R^{*}\right)-1-\left(\left(1 R^{*}, 6 S^{*}, 7 S^{*}, 9 R^{*}\right)-9\right.$-(Methoxymethyl)tricyclo[4.3.1.0,9]decan-7-yl)prop-2-yn-1-ol (34). Dess-Martin periodinane $(524.3 \mathrm{mg}, 1.24 \mathrm{mmol})$ was added to a solution of $33(200 \mathrm{mg}$, $0.95 \mathrm{mmol})$ in $\mathrm{CH}_{2} \mathrm{Cl}_{2}(10 \mathrm{~mL})$. After the addition of 1 drop of water the resulting suspension was stirred at room temperature for $15 \mathrm{~min}$ and then washed with a $1 / 1$ mixture of saturated solution of $\mathrm{Na}_{2} \mathrm{~S}_{2} \mathrm{O}_{3} /$ $\mathrm{Na}_{2} \mathrm{CO}_{3}(20 \mathrm{~mL})$. The organic layer was dried over $\mathrm{MgSO}_{4}$, filtered, and concentrated under reduced pressure. The product $\left(33^{\prime}\right)$ was obtained after filtration through a pad of silica gel as a colorless oil and directly submitted to the next step. ${ }^{1} \mathrm{H}$ NMR (500 $\left.\mathrm{MHz}, \mathrm{CDCl}_{3}\right): \delta$ $9.44(\mathrm{~s}, 1 \mathrm{H}), 3.86(\mathrm{dd}, J=10.2,1.3 \mathrm{~Hz}, 1 \mathrm{H}), 3.54(\mathrm{~d}, J=10.2 \mathrm{~Hz}, 1 \mathrm{H})$, $3.39(\mathrm{~s}, 3 \mathrm{H}), 2.66(\mathrm{ddd}, J=7.3,4.4,2.7 \mathrm{~Hz}, 1 \mathrm{H}), 2.51-2.41(\mathrm{~m}, 2 \mathrm{H})$, $1.97-1.88(\mathrm{~m}, 1 \mathrm{H}), 1.64-1.40(\mathrm{~m}, 9 \mathrm{H}), 1.37(\mathrm{dd}, J=4.9,1.3 \mathrm{~Hz}$, $1 \mathrm{H}) .{ }^{13} \mathrm{C}$ NMR $\left(126 \mathrm{MHz}, \mathrm{CDCl}_{3}\right): \delta 200.7,71.6,58.9,45.5,43.1$, 39.5 , 38.1, 30.2, 29.6, 27.8, 25.5, 24.3, 23.4. Ethynylmagnesium bromide (0.5 M in THF, $2.01 \mathrm{~mL}, 1.00 \mathrm{mmol})$ was added to a solution of aldehyde $33^{\prime}$ (190 mg, $\left.0.91 \mathrm{mmol}\right)$ in anhydrous THF $(9 \mathrm{~mL})$ at 0 ${ }^{\circ} \mathrm{C}$. After it was stirred at room temperature for $30 \mathrm{~min}$, the reaction mixture was diluted with $\mathrm{Et}_{2} \mathrm{O}(15 \mathrm{~mL})$ and quenched by the addition of saturated $\mathrm{NH}_{4} \mathrm{Cl}$ aqueous solution $(50 \mathrm{~mL})$. The aqueous layer was extracted with $\mathrm{Et}_{2} \mathrm{O}(2 \times 40 \mathrm{~mL})$, the combined organic phases were dried over $\mathrm{MgSO}_{4}$, filtered, and concentrated under reduced pressure, and the resulting crude was purified by column chromatography (cyclohexane/EtOAc 7/3) to afford 34 as a colorless oil $(208.9 \mathrm{mg}$, $0.89 \mathrm{mmol}$, yield over two steps $98 \%) .{ }^{1} \mathrm{H}$ NMR (500 $\left.\mathrm{MHz}, \mathrm{CDCl}_{3}\right)$ : $\delta 4.87(\mathrm{dd}, J=5.2,2.2 \mathrm{~Hz}, 1 \mathrm{H}), 3.81(\mathrm{~d}, J=10.4 \mathrm{~Hz}, 1 \mathrm{H}), 3.70(\mathrm{~d}, J=$ $10.3 \mathrm{~Hz}, 1 \mathrm{H}), 3.48(\mathrm{~d}, J=5.3 \mathrm{~Hz}, 1 \mathrm{H}), 3.36(\mathrm{~s}, 3 \mathrm{H}), 2.51(\mathrm{~d}, J=2.2$ $\mathrm{Hz}, 1 \mathrm{H}), 2.42$ (ddd, $J=7.7,5.3,2.4 \mathrm{~Hz}, 1 \mathrm{H}), 2.31$ (ddd, $J=7.8,6.1$, $1.9 \mathrm{~Hz}, 1 \mathrm{H}), 2.08-2.01(\mathrm{~m}, 1 \mathrm{H}), 1.86-1.79(\mathrm{~m}, 1 \mathrm{H}), 1.78-1.71(\mathrm{~m}$, $1 \mathrm{H}), 1.70-1.61(\mathrm{~m}, 1 \mathrm{H}), 1.57-1.42(\mathrm{~m}, 4 \mathrm{H}), 1.40-1.32(\mathrm{~m}, 2 \mathrm{H})$, $0.94(\mathrm{~d}, J=4.7 \mathrm{~Hz}, 1 \mathrm{H}), 0.82(\mathrm{~d}, J=4.7 \mathrm{~Hz}, 1 \mathrm{H}) .{ }^{13} \mathrm{C}$ NMR $(126$ $\left.\mathrm{MHz}, \mathrm{CDCl}_{3}\right): \delta 84.5,74.0,72.8,61.1,58.4,42.1,41.5,38.7,35.8$, 29.6, 29.1, 29.0, 25.2, 24.5, 16.4. HRMS (ESI+): $\mathrm{m} / \mathrm{z}$ calcd for $\mathrm{C}_{15} \mathrm{H}_{22} \mathrm{NaO}_{2}[\mathrm{M}+\mathrm{Na}]^{+}$, 257.1512; found, 257.1514.
Synthesis of $4 \mathrm{~d}, \mathrm{e}$. Dess-Martin periodinane (478.8 mg, 1.13 $\mathrm{mmol})$ was added to a solution of $\mathbf{4 b}, \mathbf{c}(0.87 \mathrm{mmol})$ in $\mathrm{CH}_{2} \mathrm{Cl}_{2}$ (9 $\mathrm{mL}$ ). After the addition of 1 drop of water, the resulting suspension was stirred at room temperature for $1 \mathrm{~h}$ and then washed with a $1 / 1$ mixture of a saturated solution of $\mathrm{Na}_{2} \mathrm{~S}_{2} \mathrm{O}_{3} / \mathrm{Na}_{2} \mathrm{CO}_{3}(40 \mathrm{~mL})$. The organic layer was dried over $\mathrm{MgSO}_{4}$, filtered, and concentrated under reduced pressure. The product was obtained after purification by flash chromatography (cyclohexane/EtOAc 7/3).

1-((1R*,6S*,7R*,9S*)-9-(Methoxymethyl)tricyclo[4.3.1.0 7,9$]$ deca2,4-dien-7-yl)prop-2-yn-1-one (4d). Yellow oil $(121.2 \mathrm{mg}, 0.53 \mathrm{mmol}$, yield $61 \%) .{ }^{1} \mathrm{H}$ NMR (400 MHz, $\mathrm{CDCl}_{3}$ ): $\delta 6.32$ (ddd, $J=11.4,7.4$, $1.1 \mathrm{~Hz}, 1 \mathrm{H}), 6.02$ (ddd, $J=11.2,7.7,0.9 \mathrm{~Hz}, 1 \mathrm{H}), 5.79$ (ddd, $J=11.5$, 7.4, $0.8 \mathrm{~Hz}, 1 \mathrm{H}), 5.70$ (ddd, $J=11.4,7.4,0.8 \mathrm{~Hz}, 1 \mathrm{H}), 4.02$ (dd, $J=$ 10.1, $1.5 \mathrm{~Hz}, 1 \mathrm{H}), 3.82(\mathrm{~d}, J=10.1 \mathrm{~Hz}, 1 \mathrm{H}), 3.42(\mathrm{~s}, 3 \mathrm{H}), 3.26(\mathrm{~s}$, $1 \mathrm{H}), 3.12-3.06(\mathrm{~m}, 1 \mathrm{H}), 2.91(\mathrm{t}, J=7.0 \mathrm{~Hz}, 1 \mathrm{H}), 2.06-1.98(\mathrm{~m}, 1 \mathrm{H})$, $1.85(\mathrm{dd}, J=13.2,0.6 \mathrm{~Hz}, 1 \mathrm{H}), 1.34(\mathrm{dd}, J=5.5,1.4 \mathrm{~Hz}, 1 \mathrm{H}), 1.26(\mathrm{~d}$, $J=5.5 \mathrm{~Hz}, 1 \mathrm{H}) .{ }^{13} \mathrm{C} \mathrm{NMR}\left(126 \mathrm{MHz}, \mathrm{CDCl}_{3}\right): \delta 185.7,136.9,134.8$, 125.5, 124.1, 82.0, 79.1, 71.4, 58.8, 52.3, 45.5, 41.7, 40.4, 25.6, 22.5. HRMS (ESI +$): m / z$ calcd for $\mathrm{C}_{15} \mathrm{H}_{16} \mathrm{NaO}_{2}[\mathrm{M}+\mathrm{Na}]^{+}, 251.1043$; found, 251.1035 .

1-((1R*,6S*,7R*,9S*)-9-((Benzyloxy)methyl)tricyclo[4.3.1.0 $\left.{ }^{7,9}\right]$ deca-2,4-dien-7-yl)prop-2-yn-1-one (4e). Yellow oil (174.9 mg, 0.57 mmol, yield 66\%). ${ }^{1} \mathrm{H}$ NMR (500 MHz, $\left.\mathrm{CDCl}_{3}\right): \delta 7.42-7.34(\mathrm{~m}$, 4H), 7.34-7.29 (m, 1H), 6.32 (ddq, $J=11.3,7.6,0.7 \mathrm{~Hz}, 1 \mathrm{H}), 5.97$ (ddq, $J=11.4,7.5,1.0 \mathrm{~Hz}, 1 \mathrm{H}), 5.75$ (ddd, $J=11.3,7.4,0.8 \mathrm{~Hz}, 1 \mathrm{H}$ ), $5.67(\mathrm{ddd}, J=11.4,7.3,0.8 \mathrm{~Hz}, 1 \mathrm{H}), 4.61(\mathrm{~d}, J=11.9 \mathrm{~Hz}, 1 \mathrm{H}), 4.58$ $(\mathrm{d}, J=12.0 \mathrm{~Hz}, 1 \mathrm{H}), 4.10(\mathrm{dd}, J=10.1,1.5 \mathrm{~Hz}, 1 \mathrm{H}), 3.95(\mathrm{~d}, J=10.0$ $\mathrm{Hz}, 1 \mathrm{H}), 3.11(\mathrm{~s}, 1 \mathrm{H}), 3.07(\mathrm{t}, J=7.1 \mathrm{~Hz}, 1 \mathrm{H}), 2.98(\mathrm{t}, J=7.0 \mathrm{~Hz}$, $1 \mathrm{H}), 2.02(\mathrm{dtt}, J=13.0,6.4,1.3 \mathrm{~Hz}, 1 \mathrm{H}), 1.85(\mathrm{~d}, J=14.0 \mathrm{~Hz}, 1 \mathrm{H})$, $1.35(\mathrm{dd}, J=5.6,1.4 \mathrm{~Hz}, 1 \mathrm{H}), 1.27(\mathrm{~d}, J=5.6 \mathrm{~Hz}, 1 \mathrm{H}) .{ }^{13} \mathrm{C} \mathrm{NMR}$ $\left(126 \mathrm{MHz}, \mathrm{CDCl}_{3}\right): \delta 185.7,138.5,137.0,134.7,128.4,127.8,127.6$, 125.5, 124.0, 81.7, 79.2, 73.0, 68.9, 52.6, 45.7, 41.8, 40.4, 25.5, 22.5. HRMS (ESI+): $m / z$ calcd for $\mathrm{C}_{21} \mathrm{H}_{20} \mathrm{NaO}_{2}[\mathrm{M}+\mathrm{Na}]^{+}, 327.1356$; found, 327.1363 .

Gold-Catalyzed Cyclizations of 1,6-Enynes 4d,e. Gold(I) complex B (3.1 mg, $0.002 \mathrm{mmol})^{31}$ was added to a solution of $4 \mathrm{~d}, \mathrm{e}$ $(0.1 \mathrm{mmol})$ in $\mathrm{ROH}(1 \mathrm{~mL})$ or a $2 / 1$ dioxane $/ \mathrm{H}_{2} \mathrm{O}$ mixture $(2 \mathrm{~mL})$, and the resulting suspension was stirred at room temperature for the appointed time before the addition of 1 drop of $\mathrm{Et}_{3} \mathrm{~N}$. Then the volatiles were removed under reduced pressure and purification by preparative TLC afforded the tetracyclic products $39^{\prime} / 39^{\prime \prime}$.

$\left(1 a R^{*}, 2 S *, 3 a S^{*}, 4 S *, 6 a S^{*}, 7 R^{*}\right)-1 a-(($ Benzyloxy)methyl)-7-methoxy-5-methylene-1a,2,3,3a,4,5-hexahydro-2,4-prop [1]enocyclopropa[c]pentalen-6(1H)-one $\left(39 a^{\prime}\right)$. General procedure starting from $4 \mathrm{e}$ and methanol. Reaction time: $1 \mathrm{~h}$. Purification: cyclohexane/EtOAc 95/5 (eluted three times). White solid (15.1 mg, yield $45 \%)$. Mp: $59-61{ }^{\circ} \mathrm{C} .{ }^{1} \mathrm{H}$ NMR (400 $\mathrm{MHz}, \mathrm{CDCl}_{3}$ ): $\delta 7.40-$ $7.28(\mathrm{~m}, 5 \mathrm{H}), 6.14-6.07(\mathrm{~m}, 2 \mathrm{H}), 5.72(\mathrm{dd}, J=11.8,7.2 \mathrm{~Hz}, 1 \mathrm{H})$, $5.33(\mathrm{dd}, J=2.2,0.6 \mathrm{~Hz}, 1 \mathrm{H}), 4.58(\mathrm{~d}, J=11.9 \mathrm{~Hz}, 1 \mathrm{H}), 4.44(\mathrm{~d}, J=$ $12.0 \mathrm{~Hz}, 1 \mathrm{H}), 3.82(\mathrm{ddd}, J=7.3,3.1,0.7 \mathrm{~Hz}, 1 \mathrm{H}), 3.68(\mathrm{dd}, J=10.0$, $1.9 \mathrm{~Hz}, 1 \mathrm{H}), 3.54-3.48(\mathrm{~m}, 1 \mathrm{H}), 3.44(\mathrm{~s}, 3 \mathrm{H}), 3.07(\mathrm{~d}, J=9.9 \mathrm{~Hz}$, $1 \mathrm{H}), 3.03(\mathrm{t}, J=6.6 \mathrm{~Hz}, 1 \mathrm{H}), 2.89(\mathrm{dd}, J=9.3,7.2 \mathrm{~Hz}, 1 \mathrm{H}), 2.74(\mathrm{~d}, J$ $=13.9 \mathrm{~Hz}, 1 \mathrm{H}), 1.87(\mathrm{~d}, J=5.7 \mathrm{~Hz}, 1 \mathrm{H}), 1.68(\mathrm{dtd}, J=13.9,6.9,1.7$ $\mathrm{Hz}, 1 \mathrm{H}), 1.52(\mathrm{dd}, J=5.8,1.8 \mathrm{~Hz}, 1 \mathrm{H}) .{ }^{13} \mathrm{C}$ NMR $(101 \mathrm{MHz}$, $\left.\mathrm{CDCl}_{3}\right): \delta 201.7,147.6,140.4,138.3,128.4,127.7,127.7,127.1,118.0$, 82.7, 73.1, 70.5, 56.6, 52.7, 47.3, 45.6, 42.4, 42.0, 31.5, 22.4. HRMS (ESI+): $m / z$ calcd for $\mathrm{C}_{22} \mathrm{H}_{24} \mathrm{NaO}_{3}[\mathrm{M}+\mathrm{Na}]^{+}, 359.1618$; found, 359.1617 .

$\left(1 a R^{*}, 2 R^{*}, 3 a S^{*}, 4 S^{*}, 6 a S^{*}, 9 S^{*}\right)-1 a-(($ Benzyloxy)methyl)-9-methoxy-5-methylene-1a,2,3,3a,4,5-hexahydro-4,2-prop [1]enocyclopropa[c]pentalen-6(1H)-one $\left(39 a^{\prime \prime}\right)$. General procedure starting from $4 \mathrm{e}$ and methanol. Reaction time: $1 \mathrm{~h}$. Purification: cyclohexane/EtOAc $95 / 5$ (eluted three times). White solid ( $9.7 \mathrm{mg}$, yield $29 \%$ ). Mp: $70-72{ }^{\circ} \mathrm{C} .{ }^{1} \mathrm{H}$ NMR (400 $\left.\mathrm{MHz}, \mathrm{CDCl}_{3}\right): \delta 7.40-$ $7.29(\mathrm{~m}, 5 \mathrm{H}), 5.92(\mathrm{dd}, J=1.4,0.7 \mathrm{~Hz}, 1 \mathrm{H}), 5.43(\mathrm{ddt}, J=12.8,4.7$, $1.9 \mathrm{~Hz}, 1 \mathrm{H}), 5.34(\mathrm{ddd}, J=12.9,4.9,0.9 \mathrm{~Hz}, 1 \mathrm{H}), 5.28(\mathrm{dd}, J=1.3,0.7$ $\mathrm{Hz}, 1 \mathrm{H}), 4.60(\mathrm{~d}, J=11.9 \mathrm{~Hz}, 1 \mathrm{H}), 4.41(\mathrm{~d}, J=11.9 \mathrm{~Hz}, 1 \mathrm{H}), 3.90(\mathrm{t}, J$ $=4.0 \mathrm{~Hz}, 1 \mathrm{H}), 3.60(\mathrm{t}, J=6.3 \mathrm{~Hz}, 1 \mathrm{H}), 3.52(\mathrm{dd}, J=10.4,1.8 \mathrm{~Hz}$, $1 \mathrm{H}), 3.38(\mathrm{~s}, 3 \mathrm{H}), 3.17(\mathrm{~d}, J=10.4 \mathrm{~Hz}, 1 \mathrm{H}), 2.83(\mathrm{dt}, J=8.0,2.6 \mathrm{~Hz}$, $1 \mathrm{H}), 2.76(\mathrm{t}, J=7.4 \mathrm{~Hz}, 1 \mathrm{H}), 2.29(\mathrm{~d}, J=14.4 \mathrm{~Hz}, 1 \mathrm{H}), 1.77(\mathrm{~d}, J=$ 
$5.4 \mathrm{~Hz}, 1 \mathrm{H}), 1.68-1.61(\mathrm{~m}, 1 \mathrm{H}), 1.34(\mathrm{dd}, J=5.5,1.7 \mathrm{~Hz}, 1 \mathrm{H}) .{ }^{13} \mathrm{C}$ NMR (101 MHz, $\left.\mathrm{CDCl}_{3}\right): \delta 202.1,149.7,138.1,131.5,128.4,127.9$, 127.8, 124.1, 115.5, 78.2, 73.1, 69.3, 56.9, 47.5, 47.1, 44.8, 43.6, 43.2, 26.7, 21.0. HRMS (ESI+): $m / z$ calcd for $\mathrm{C}_{22} \mathrm{H}_{24} \mathrm{NaO}_{3}[\mathrm{M}+\mathrm{Na}]^{+}$, 359.1618; found, 359.1616 .

$(1 a R *, 2 S *, 3 a S *, 4 S *, 6 a S *, 7 R *)-7-($ Allyloxy) $-1 a-(($ benzyloxy)methyl)-5-methylene-1a,2,3,3a,4,5-hexahydro-2,4-prop[1]enocyclopropa[c]pentalen-6(1H)-one $\left(39 b^{\prime}\right)$. General procedure starting from $4 \mathrm{e}$ and allyl alcohol. Reaction time: $1.5 \mathrm{~h}$. Purification: cyclohexane/EtOAc $95 / 5$ (eluted three times). White solid $(15.9 \mathrm{mg}$, yield $44 \%)$. Mp: $44-46{ }^{\circ} \mathrm{C} .{ }^{1} \mathrm{H}$ NMR $\left(400 \mathrm{MHz}, \mathrm{CDCl}_{3}\right): \delta 7.39-$ $7.28(\mathrm{~m}, 5 \mathrm{H}), 6.13-6.07(\mathrm{~m}, 2 \mathrm{H}), 5.98(\mathrm{ddt}, J=17.2,10.3,5.6 \mathrm{~Hz}$, $1 \mathrm{H}), 5.68(\mathrm{dd}, J=11.7,7.3 \mathrm{~Hz}, 1 \mathrm{H}), 5.34(\mathrm{dq}, J=17.1,1.4 \mathrm{~Hz}, 1 \mathrm{H})$, $5.31-5.29(\mathrm{~m}, 1 \mathrm{H}), 5.23(\mathrm{dq}, J=10.4,1.4 \mathrm{~Hz}, 1 \mathrm{H}), 4.58(\mathrm{~d}, J=11.9$ $\mathrm{Hz}, 1 \mathrm{H}), 4.44(\mathrm{~d}, J=11.8 \mathrm{~Hz}, 1 \mathrm{H}), 4.15(\mathrm{ddt}, J=12.6,5.5,1.5 \mathrm{~Hz}$, $1 \mathrm{H}), 4.07$ (ddt, $J=12.6,5.7,1.4 \mathrm{~Hz}, 1 \mathrm{H}), 3.97$ (ddd, $J=7.3,3.1,0.6$ $\mathrm{Hz}, 1 \mathrm{H}), 3.68(\mathrm{dd}, J=10.0,1.8 \mathrm{~Hz}, 1 \mathrm{H}), 3.50(\mathrm{dq}, J=9.2,2.5 \mathrm{~Hz}$, $1 \mathrm{H}), 3.06(\mathrm{~d}, J=10.0 \mathrm{~Hz}, 1 \mathrm{H}), 3.06(\mathrm{t}, J=6.7 \mathrm{~Hz}, 1 \mathrm{H}), 2.90(\mathrm{dd}, J=$ 9.3, 7.2 Hz, 1H), $2.80(\mathrm{~d}, J=13.9 \mathrm{~Hz}, 1 \mathrm{H}), 1.86(\mathrm{~d}, J=5.7 \mathrm{~Hz}, 1 \mathrm{H})$, $1.69(\mathrm{dtd}, J=13.9,6.9,1.7 \mathrm{~Hz}, 1 \mathrm{H}), 1.52(\mathrm{dd}, J=5.7,1.8 \mathrm{~Hz}, 1 \mathrm{H}) .{ }^{13} \mathrm{C}$ $\operatorname{NMR}\left(101 \mathrm{MHz}, \mathrm{CDCl}_{3}\right): \delta 201.7,147.7,140.3,138.3,135.1,128.4$, 127.7, 127.7, 127.3, 118.0, 117.2, 80.1, 73.1, 70.5, 69.7, 52.8, 47.3, 46.0, 42.4, 42.0, 31.6, 22.4. HRMS (ESI+): $m / z$ calcd for $\mathrm{C}_{24} \mathrm{H}_{26} \mathrm{NaO}_{3}[\mathrm{M}+$ $\mathrm{Na}]^{+}, 385.1774$; found, 385.1780.

$\left(1 a R^{*}, 2 R * 3 a S^{*}, 4 S *, 6 a S *, 9 S *\right)-9$-(Allyloxy)-1a-((benzyloxy)methyl)-5-methylene-1a,2,3,3a,4,5-hexahydro-4,2-prop [1]enocyclopropa[c]pentalen-6(1H)-one $\left(39 b^{\prime \prime}\right)$. General procedure starting from $4 \mathrm{e}$ and allyl alcohol. Reaction time: $1.5 \mathrm{~h}$. Purification: cyclohexane/EtOAc $95 / 5$ (eluted three times). White solid $(15.1 \mathrm{mg}$, yield $42 \%)$. Mp: $67-69{ }^{\circ} \mathrm{C} .{ }^{1} \mathrm{H}$ NMR $\left(400 \mathrm{MHz}, \mathrm{CDCl}_{3}\right): \delta 7.39-$ $7.29(\mathrm{~m}, 5 \mathrm{H}), 6.00-5.86(\mathrm{~m}, 1 \mathrm{H}), 5.93-5.91(\mathrm{~m}, 1 \mathrm{H}), 5.42(\mathrm{ddt}, J=$ $12.8,4.6,1.8 \mathrm{~Hz}, 1 \mathrm{H}), 5.37-5.31(\mathrm{~m}, 1 \mathrm{H}), 5.29-5.27(\mathrm{~m}, 1 \mathrm{H}), 5.28$ $(\mathrm{dq}, J=17.2,1.6 \mathrm{~Hz}, 1 \mathrm{H}), 5.18(\mathrm{dq}, J=10.3,1.4 \mathrm{~Hz}, 1 \mathrm{H}), 4.57(\mathrm{~d}, J=$ $11.8 \mathrm{~Hz}, 1 \mathrm{H}), 4.41(\mathrm{~d}, J=11.8 \mathrm{~Hz}, 1 \mathrm{H}), 4.13-4.04(\mathrm{~m}, 2 \mathrm{H}), 3.99$ $(\mathrm{ddt}, J=12.8,5.8,1.4 \mathrm{~Hz}, 1 \mathrm{H}), 3.61(\mathrm{t}, J=6.0 \mathrm{~Hz}, 1 \mathrm{H}), 3.52(\mathrm{dd}, J=$ $10.4,1.8 \mathrm{~Hz}, 1 \mathrm{H}), 3.16(\mathrm{~d}, J=10.4 \mathrm{~Hz}, 1 \mathrm{H}), 2.84-2.79(\mathrm{~m}, 1 \mathrm{H}), 2.77$ $(\mathrm{td}, J=7.1,1.1 \mathrm{~Hz}, 1 \mathrm{H}), 2.36(\mathrm{~d}, J=14.4 \mathrm{~Hz}, 1 \mathrm{H}), 1.77(\mathrm{~d}, J=5.4 \mathrm{~Hz}$, $1 \mathrm{H}), 1.71-1.59(\mathrm{~m}, 1 \mathrm{H}), 1.33(\mathrm{dd}, J=5.4,1.7 \mathrm{~Hz}, 1 \mathrm{H}) .{ }^{13} \mathrm{C}$ NMR $\left(101 \mathrm{MHz}, \mathrm{CDCl}_{3}\right): \delta 202.1,149.7,138.1,135.1,131.5,128.4,127.9$, 127.7, 124.5, 117.0, 115.5, 76.0, 73.2, 70.2, 69.5, 47.4, 47.1, 44.9, 43.8, 43.6, 26.8, 21.0. HRMS (ESI+): $m / z$ calcd for $\mathrm{C}_{24} \mathrm{H}_{26} \mathrm{NaO}_{3}[\mathrm{M}+$ $\mathrm{Na}]^{+}, 385.1774$; found, 385.1780.

$\left(1 a R^{*}, 2 S *, 3 a S^{*}, 4 S *, 6 a S *, 7 R *\right)-7-H y d r o x y-1 a-(m e t h o x y m e t h y l)-$ 5-methylene-1a,2,3,3a,4,5-hexahydro-2,4-prop[1]enocyclopropa[c]pentalen-6(1H)-one $\left(39 c^{\prime}\right)$ and $\left(1 a R^{*}, 2 R^{*}, 3 a S^{*}, 4 S^{*}, 6 a S^{*}, 9 S^{*}\right)-9$ hydroxy-1a-(methoxymethyl)-5-methylene-1a,2,3,3a,4,5-hexahydro-4,2-prop[1]enocyclopropa[c]pentalen-6(1H)-one (39c"). General procedure starting from $4 \mathbf{d}$ and water. Reaction time: $3 \mathrm{~h}$. Purification: cyclohexane/EtOAc 6/4 (eluted twice). Colorless oil (15.5 mg, yield 63\%). 39 $\mathrm{c}^{\prime}: 39 \mathrm{c}^{\prime \prime}=1: 2 .{ }^{1} \mathrm{H}$ NMR (400 MHz, $\left.\mathrm{CDCl}_{3}\right)$ : $39 \mathrm{c}^{\prime}, \delta 6.17(\mathrm{ddd}, J=11.6,6.8,1.6 \mathrm{~Hz}, 1 \mathrm{H}), 6.11(\mathrm{dd}, J=2.5,0.6 \mathrm{~Hz}$, $1 \mathrm{H}), 5.82(\mathrm{dd}, J=11.6,7.3 \mathrm{~Hz}, 1 \mathrm{H}), 5.40-5.32(\mathrm{~m}, 1 \mathrm{H}), 4.38(\mathrm{dd}, J=$ 7.3, $3.1 \mathrm{~Hz}, 1 \mathrm{H}), 3.59$ (dd, $J=10.1,1.9 \mathrm{~Hz}, 1 \mathrm{H}), 3.47(\mathrm{dd}, J=9.3,2.7$ $\mathrm{Hz}, 1 \mathrm{H}), 3.35(\mathrm{~s}, 3 \mathrm{H}), 3.05-2.91(\mathrm{~m}, 2 \mathrm{H}), 2.93(\mathrm{~d}, J=10.1 \mathrm{~Hz}, 1 \mathrm{H})$, $2.75(\mathrm{~d}, J=14.2 \mathrm{~Hz}, 1 \mathrm{H}), 1.86(\mathrm{~d}, J=5.7 \mathrm{~Hz}, 1 \mathrm{H}), 1.79-1.66(\mathrm{~m}$, $1 \mathrm{H}), 1.50(\mathrm{dd}, J=5.8,1.9 \mathrm{~Hz}, 1 \mathrm{H}) ; 39 \mathrm{c}^{\prime \prime}, \delta 5.94(\mathrm{dd}, J=1.4,0.6 \mathrm{~Hz}$, $1 \mathrm{H}), 5.49(\mathrm{ddt}, J=12.7,4.9,2.0 \mathrm{~Hz}, 1 \mathrm{H}), 5.39-5.33(\mathrm{~m}, 1 \mathrm{H}), 5.30$ $(\mathrm{dd}, J=1.4,0.6 \mathrm{~Hz}, 1 \mathrm{H}), 4.57(\mathrm{t}, J=4.1 \mathrm{~Hz}, 1 \mathrm{H}), 3.62(\mathrm{t}, J=6.6 \mathrm{~Hz}$, $1 \mathrm{H}), 3.45(\mathrm{dd}, J=10.5,1.8 \mathrm{~Hz}, 1 \mathrm{H}), 3.33(\mathrm{~s}, 3 \mathrm{H}), 3.03(\mathrm{~d}, J=10.5 \mathrm{~Hz}$, $1 \mathrm{H}), 2.79(\mathrm{td}, J=7.2,1.2 \mathrm{~Hz}, 1 \mathrm{H}), 2.67(\mathrm{dt}, J=8.0,2.7 \mathrm{~Hz}, 1 \mathrm{H}), 2.30$ $(\mathrm{d}, J=14.5 \mathrm{~Hz}, 1 \mathrm{H}), 1.76(\mathrm{~d}, J=5.5 \mathrm{~Hz}, 1 \mathrm{H}), 1.74-1.64(\mathrm{~m}, 1 \mathrm{H})$, $1.32(\mathrm{dd}, J=5.5,1.8 \mathrm{~Hz}, 1 \mathrm{H}) .{ }^{13} \mathrm{C}$ NMR $\left(101 \mathrm{MHz}, \mathrm{CDCl}_{3}\right): 39 \mathrm{c}^{\prime}+$ $39 \mathrm{c}^{\prime \prime}, \delta 202.0,201.6,149.4,147.5,139.8,131.2,129.2,126.0,118.4$, $115.7,74.5,72.8,71.7,69.0,58.9,58.8,52.3,48.2,47.4,47.2,46.7$, 44.7, 43.5, 42.3, 41.9, 32.1, 29.7, 26.4, 21.9, 20.9. HRMS (ESI+): $\mathrm{m} / z$ calcd for $\mathrm{C}_{15} \mathrm{H}_{18} \mathrm{NaO}_{3}[\mathrm{M}+\mathrm{Na}]^{+}$, 269.1148; found, 269.1139 .

$(1 a R *, 2 S *, 3 a S *, 4 S *, 6 a S *, 7 R *)-1 a-(($ Benzyloxy)methyl)-7-hydroxy-5-methylene-1a,2,3,3a,4,5-hexahydro-2,4-prop [1]enocyclopropa[c]pentalen-6(1H)-one $\left(39 d^{\prime}\right)$ and $\left(1 a R^{*}, 2 R^{*}, 3 a S^{*}, 4\right.$ $\left.S^{*}, 6 a S^{*}, 9 S^{*}\right)-1 a-(($ benzyloxy)methyl)-9-hydroxy-5-methylene1a,2,3,3a,4,5-hexahydro-4,2-prop[1]enocyclopropa[c]pentalen-
$6(1 H)$-one $\left(39 d^{\prime \prime}\right)$. General procedure starting from $4 \mathrm{e}$ and water. Reaction time: $5 \mathrm{~h}$. Purification: cyclohexane/EtOAc 6/4 (eluted twice). Colorless oil (18.6 mg, yield 58\%). 39d':39d" = 1:4. ${ }^{1} \mathrm{H}$ NMR $\left(500 \mathrm{MHz}, \mathrm{CDCl}_{3}\right): 39 \mathrm{~d}^{\prime} \delta 7.38-7.31(\mathrm{~m}, 5 \mathrm{H}), 6.10(\mathrm{dd}, J=2.5,0.6$ $\mathrm{Hz}, 1 \mathrm{H}), 6.08$ (ddd, $J=11.6,6.9,1.6 \mathrm{~Hz}, 1 \mathrm{H}), 5.76(\mathrm{dd}, J=11.7,7.3$ $\mathrm{Hz}, 1 \mathrm{H}), 5.38-5.31(\mathrm{~m}, 1 \mathrm{H}), 4.56(\mathrm{~d}, J=11.9 \mathrm{~Hz}, 1 \mathrm{H}), 4.44(\mathrm{~d}, J=$ $11.9 \mathrm{~Hz}, 1 \mathrm{H}), 4.38-4.34(\mathrm{~m}, 1 \mathrm{H}), 3.68(\mathrm{dd}, J=10.0,1.8 \mathrm{~Hz}, 1 \mathrm{H})$, $3.47(\mathrm{dq}, J=9.2,2.1 \mathrm{~Hz}, 1 \mathrm{H}), 3.08(\mathrm{~d}, J=9.8 \mathrm{~Hz}, 1 \mathrm{H}), 3.05(\mathrm{~d}, J=6.7$ $\mathrm{Hz}, 1 \mathrm{H}), 2.96$ (dd, $J=9.1,7.2 \mathrm{~Hz}, 1 \mathrm{H}), 2.74(\mathrm{~d}, J=14.1 \mathrm{~Hz}, 1 \mathrm{H}), 1.87$ $(\mathrm{d}, J=5.8 \mathrm{~Hz}, 1 \mathrm{H}), 1.79-1.74(\mathrm{~m}, 1 \mathrm{H}), 1.52(\mathrm{dd}, J=5.7,1.8 \mathrm{~Hz}, 1 \mathrm{H})$; $39 \mathrm{~d}^{\prime \prime}, \delta 7.39-7.29(\mathrm{~m}, 6 \mathrm{H}), 5.94(\mathrm{dd}, J=1.4,0.6 \mathrm{~Hz}, 1 \mathrm{H}), 5.42(\mathrm{ddt}, J$ $=12.7,4.7,1.9 \mathrm{~Hz}, 1 \mathrm{H}), 5.34(\mathrm{ddt}, J=12.7,5.2,0.9 \mathrm{~Hz}, 1 \mathrm{H}), 5.29(\mathrm{dd}$, $J=1.3,0.6 \mathrm{~Hz}, 1 \mathrm{H}), 4.55(\mathrm{~d}, J=12.0 \mathrm{~Hz}, 1 \mathrm{H}), 4.52(\mathrm{~s}, 1 \mathrm{H}), 4.43(\mathrm{~d}, J$ $=11.8 \mathrm{~Hz}, 1 \mathrm{H}), 3.64-3.59(\mathrm{~m}, 1 \mathrm{H}), 3.53(\mathrm{dd}, J=10.5,1.7 \mathrm{~Hz}, 1 \mathrm{H})$, $3.20(\mathrm{~d}, J=10.5 \mathrm{~Hz}, 1 \mathrm{H}), 2.82-2.77(\mathrm{~m}, 1 \mathrm{H}), 2.73-2.69(\mathrm{~m}, 1 \mathrm{H})$, $2.29(\mathrm{~d}, J=14.5 \mathrm{~Hz}, 1 \mathrm{H}), 1.77(\mathrm{~d}, J=5.4 \mathrm{~Hz}, 1 \mathrm{H}), 1.69$ (dddd, $J=$ $14.8,8.3,7.1,1.5 \mathrm{~Hz}, 1 \mathrm{H}), 1.34$ (dd, $J=5.5,1.7 \mathrm{~Hz}, 1 \mathrm{H}) .{ }^{13} \mathrm{C}$ NMR $\left(126 \mathrm{MHz}, \mathrm{CDCl}_{3}\right): 39 \mathrm{~d}^{\prime}+39 \mathrm{~d}^{\prime \prime}, \delta 201.9,149.4,139.9,138.0,131.1$, $129.8,128.4,128.4,127.8,127.74,127.7,127.7,127.7,126.1,118.3$, 115.7, 74.5, 73.2, 73.1, 70.5, 69.5, 69.1, 52.5, 48.2, 47.4, 47.3, 46.8, 45.0, 43.5, 42.4, 42.0, 32.1, 26.4, 22.1, 21.0 (2 peaks missing due to overlapping). HRMS (ESI+): $m / z$ calcd for $\mathrm{C}_{21} \mathrm{H}_{22} \mathrm{NaO}_{3}[\mathrm{M}+\mathrm{Na}]^{+}$, 345.1461; found, 345.1459.

\section{ASSOCIATED CONTENT}

\section{Supporting Information}

The Supporting Information is available free of charge on the ACS Publications website at DOI: 10.1021/acs.joc.6b01607.

Spectral data for all new compounds and Cartesian coordinates of the optimized structures (PDF)

X-ray crystallography data for compound 19 (CIF)

$\mathrm{X}$-ray crystallography data for compound 32a (CIF)

\section{AUTHOR INFORMATION}

\section{Corresponding Author}

*E-mail for A.M.E.: aechavarren@iciq.es.

\section{Notes}

The authors declare no competing financial interest.

\section{ACKNOWLEDGMENTS}

We thank MINECO (Severo Ochoa Excellence Accreditation 2014-2018 (SEV-2013-0319), project CTQ2013-42106-P), the European Research Council (Advanced Grant No. 321066), the AGAUR (2014 SGR 818), and the ICIQ Foundation. We also thank the ICIQ X-ray diffraction unit for the structures of 19 and $32 a$.

\section{REFERENCES}

(1) Dong, M.; Cong, B.; Yu, S.-H.; Sauriol, F.; Huo, C.-H.; Shi, Q.W.; Gu, Y.-C.; Zamir, L. O.; Kiyota, H. Org. Lett. 2008, 10, 701-704.

(2) Magauer, T.; Mulzer, J.; Tiefenbacher, K. Org. Lett. 2009, 11, 5306-5309.

(3) Nicolaou, K. C.; Ding, H.; Richard, J.-A.; Chen, D. Y.-K. J. Am. Chem. Soc. 2010, 132, 3815-3818.

(4) Peixoto, P. A.; Severin, R.; Tseng, C.-C.; Chen, D. Y.-K. Angew. Chem., Int. Ed. 2011, 50, 3013-3016.

(5) Xu, W.; Wu, S.; Zhou, L.; Liang, G. Org. Lett. 2013, 15, 19781981.

(6) Michels, T. D.; Dowling, M. S.; Vanderwal, C. D. Angew. Chem., Int. Ed. 2012, 51, 7572-7576.

(7) De, S.; Misra, S.; Rigby, J. H. Org. Lett. 2015, 17, 3230-3232.

(8) Sanogo, Y.; Allievi, L.; Lecourt, C.; Dhambri, S.; Ardisson, J.; Sorin, G.; Lannou, M.-I. Tetrahedron 2016, 72, 3369-3377.

(9) Fürstner, A. Chem. Soc. Rev. 2009, 38, 3208-3221.

(10) Obradors, C.; Echavarren, A. M. Acc. Chem. Res. 2014, 47, 902912. 
(11) Fensterbank, L.; Malacria, M. Acc. Chem. Res. 2014, 47, 953965.

(12) Dorel, R.; Echavarren, A. M. Chem. Rev. 2015, 115, 9028-9072.

(13) Nieto-Oberhuber, C.; Muñoz, M. P.; Buñuel, E.; Nevado, C.; Cárdenas, D. J.; Echavarren, A. M. Angew. Chem., Int. Ed. 2004, 43, 2402-2406.

(14) Buzas, A. K.; Istrate, F. M.; Gagosz, F. Angew. Chem., Int. Ed. 2007, 46, 1141-1144.

(15) Nieto-Oberhuber, C.; Muñoz, M. P.; López, S.; Jiménez-Núñez, E.; Nevado, C.; Herrero-Gómez, E.; Raducan, M.; Echavarren, A. M. Chem. - Eur. J. 2006, 12, 1677-1693; Corrigendum: Chem. - Eur. J. 2008, 14, 5096.

(16) Martínez, A.; García-García, P.; Fernández-Rodríguez, M. A.; Rodríguez, F.; Sanz, R. Angew. Chem., Int. Ed. 2010, 49, 4633-4637.

(17) Toselli, N.; Martin, D.; Achard, M.; Tenaglia, A.; Bürgi, T.; Buono, G. Adv. Synth. Catal. 2008, 350, 280-286.

(18) Hilt, G.; Paul, A.; Hengst, C. Synthesis 2009, 2009, 3305-3310.

(19) Teles, J. H.; Brode, S.; Chabanas, M. Angew. Chem., Int. Ed. 1998, 37, 1415-1418.

(20) Mizushima, E.; Sato, K.; Hayashi, T.; Tanaka, M. Angew. Chem., Int. Ed. 2002, 41, 4563-4565.

(21) Marion, N.; Ramón, R. S.; Nolan, S. P. J. Am. Chem. Soc. 2009, $131,448-449$.

(22) Leyva, A.; Corma, A. J. Org. Chem. 2009, 74, 2067-2074.

(23) Chen, G.-Q.; Fang, W.; Wei, Y.; Tang, X.-Y.; Shi, M. Chem. Sci. 2016, 7, 4318-4328.

(24) CCDC 1489805 (19) and CCDC 1489806 (32a) contain supplementary crystallographic data for this paper. These data can be obtained free of charge from the Cambridge Crystallographic Data Centre via www.ccdc.cam.ac.uk/data_request/cif.

(25) Aldehydes $9 \mathbf{b}, \mathbf{c}$ were prepare following a route analogous to that for the preparation of $9 a$ shown in Scheme 2. See the Experimental Section.

(26) See the Supporting Information for details.

(27) For a related generation of $\alpha, \beta$-unsaturated gold(I) carbenes, see: (a) Jiménez-Núñez, E.; Raducan, M.; Lauterbach, T.; Molawi, K.; Solorio, C. R.; Echavarren, A. M. Angew. Chem., Int. Ed. 2009, 48, 6152-6155. (b) Carreras, J.; Livendahl, M.; McGonigal, P. R.; Echavarren, A. M. Angew. Chem., Int. Ed. 2014, 53, 4896-4899.

(28) For a mechanistic picture of the gold-catalyzed cyclization see the Supporting Information.

(29) Prepared according to: Najdi, S. D.; Olmstead, M. M.; Schore, N. E. J. Organomet. Chem. 1992, 431, 335-358.

(30) $\mathbf{5 b}$ was prepared according to: Wu, Y.; Huang, J.-H.; Shen, X.; Hu, Q.; Tang, C.-J.; Li, L. Org. Lett. 2002, 4, 2141-2144.

(31) Catalyst B was prepared according to: Homs, A.; Obradors, C.; Lebøuf, D.; Echavarren, A. M. Adv. Synth. Catal. 2014, 356, 221-228.

(32) Prepared according to: Takimoto, M.; Usami, S.; Hou, Z. J. Am. Chem. Soc. 2009, 131, 18266-18268.

(33) This product decomposes under ambient conditions and therefore either was directly used in the next step or was stored under argon in darkness at $-5{ }^{\circ} \mathrm{C}$. 